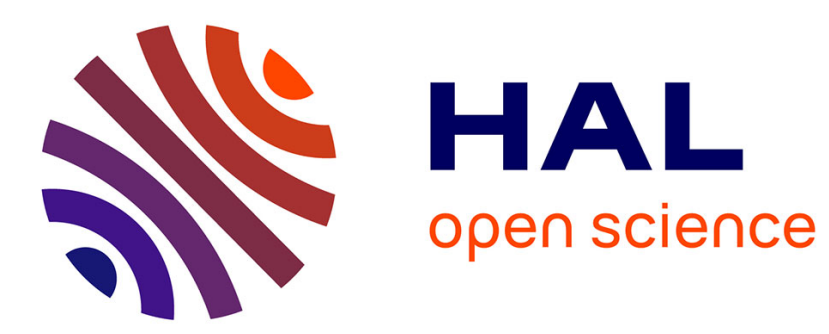

\title{
Pressure Relaxation Procedures for Multiphase Compressible Flows
}

Marie-Hélène Lallemand, Richard Saurel

\section{To cite this version:}

Marie-Hélène Lallemand, Richard Saurel. Pressure Relaxation Procedures for Multiphase Compressible Flows. [Research Report] RR-4038, INRIA. 2000. inria-00072600

\section{HAL Id: inria-00072600 \\ https://hal.inria.fr/inria-00072600}

Submitted on 24 May 2006

HAL is a multi-disciplinary open access archive for the deposit and dissemination of scientific research documents, whether they are published or not. The documents may come from teaching and research institutions in France or abroad, or from public or private research centers.
L'archive ouverte pluridisciplinaire HAL, est destinée au dépôt et à la diffusion de documents scientifiques de niveau recherche, publiés ou non, émanant des établissements d'enseignement et de recherche français ou étrangers, des laboratoires publics ou privés. 
INSTITUT NATIONAL DE RECHERCHE EN INFORMATIQUE ET EN AUTOMATIQUE

\section{Pressure relaxation procedures for multiphase compressible flows}

Marie-Hélène Lallemand — Richard Saurel

$\mathbf{N}^{\circ} \mathbf{4 0 3 8}$

Octobre 2000

THÈME 4 



\title{
Pressure relaxation procedures for multiphase compressible flows
}

\author{
Marie-Hélène Lallemand* Richard Saurel $^{\dagger}$ \\ Thème 4 - Simulation et optimisation \\ de systèmes complexes \\ Projet M3N \\ Rapport de recherche $\mathrm{n}^{\circ} 4038$ - Octobre 2000 - 31 pages
}

\begin{abstract}
Multiphase compressible flow models are usefull for the numerical simulation of a wide range of applications: interface problems, detonation physics, shock waves in mixtures, cavitation flows etc. The numerical solution of such models involves several ingredients. One of those ingredients is the instantaneous pressure relaxation process and is of particular importance.

In this report, we present and compare existing and new pressure relaxation procedures in terms of both accuracy and computational efficiency. Among those procedures we enhance one which seems to be particularly well suited for problems with large pressure variations between two phases. Moreover, it deals with general equations of state (EOS). Some tests are provided to illustrate those comparisons.
\end{abstract}

Key-words: Hyperbolic multiphase models, Riemann solvers, Finite Volume Method, Pressure relaxation.

* Marie-Helene.Lallemand@inria.fr - Projet M3N et action SMASH

$\dagger$ richard@iusti.univ-mrs.fr - Action SMASH et I.U.S.T.I.: Technopôle de Château-Gombert - 5, rue Enrico Fermi - 13453 Marseille Cédex 13 


\section{Procédures de relaxation des pressions pour les écoulements compressibles multiphasiques}

Résumé : Les modèles d'écoulements de fluides compressibles multiphasiques sont utiles pour la simulation numérique d'une grande variété d'applications: problèmes à interface, physique détonique, ondes de choc dans les mélanges, écoulements avec cavitation etc. Parmi les ingrédients qui composent la procédure de résolution numérique de ces modèles, on en retient un qui a une importance particulière: le processus de relaxation instantanée de pression.

Dans ce rapport, on présente et compare différentes procédures de relaxation instantanée de pression déjà existantes et nouvelles, en termes de précision et efficacité numériques. Parmi ces procédures, on présente une nouvelle approche qui semble particulièrement adaptée aux problèmes où la variation de pression entre chaque phase est grande. D'autre part, elle peut être utilisée pour des équations d'état générales. Ceci est illustré numériquement par quelques cas tests représentatifs.

Mots-clés : Modèles hyperboliques multiphasiques, Solveurs de Riemann, Méthodes de volumes finis, Relaxation instantanée de pression 


\section{Contents}

1 Introduction $\quad 4$

2 The 1-D two-phase model 4

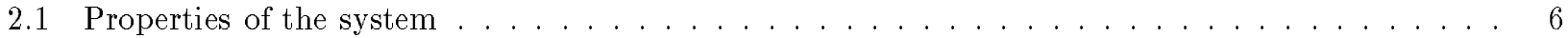

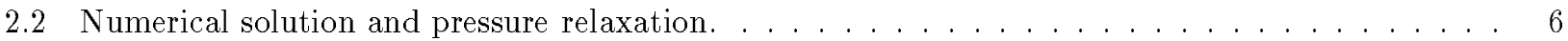

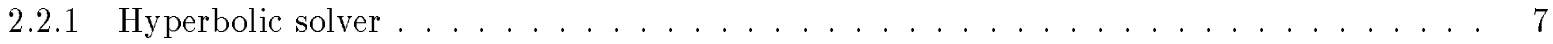

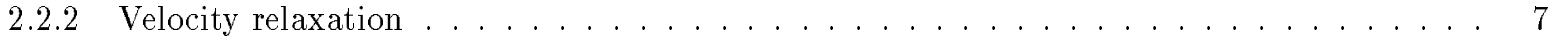

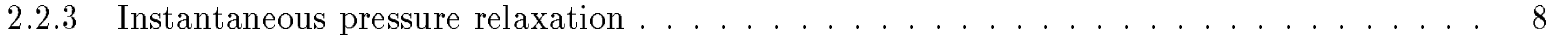

2.2 .4 A direct solution procedure for low pressure variation . . . . . . . . . . . . . . 10

2.2 .5 Iterative relaxation procedures for high pressure variation $\ldots \ldots \ldots \ldots \ldots$

3 Numerical tests 21

3.1 Water faucet test . . . . . . . . . . . . . . . . . . . . . 21

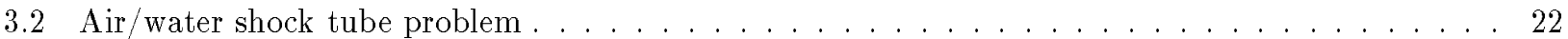

4 Conclusion 


\section{Introduction}

Recently, a new multiphase model has been proposed. This unconditionally hyperbolic model is able to solve physical situations for which the other models fail [11]. As a matter of fact, it is able to:

- solve classical multiphase flows (bubbly flows, flows with droplets, etc.) as well as interface problems. Due to a unique formulation of the whole system, this is realized by solving the whole set of equations with the same numerical method at each mesh node. Moreover, this model is the only one that ensures energy conservation at the interface leading therefore to a proper and accurate solution of the interface conditions;

- solve strong shocks in compressible mixtures (metallic alloys, powders) and is also able to solve detonation waves in condensed materials. Indeed, classical models are based on the Euler equations closed by a mixture EOS. But this mixture EOS relies on unphysical equilibrium assumption such as temperature or density equality between phases. The new model allows the determination of all thermodynamic variables of each phase, and consequently makes use of pure material EOS without using a mixture EOS;

- deal with situations where interfaces appear dynamically. For instance, in a cavitating flow, the initial pure liquid transforms partially to vapour during the rarefaction waves propagation. Cavitation pockets may be formed corresponding to new interfaces in the flow. Classical methods need the initial knowledge of those interfaces. The new model is able to make them appear dynamically.

Those important features are detailed in [9].

The numerical solution of that model requires several ingredients. The numerical method involves a non conservative hyperbolic solver, an instantaneous velocity relaxation procedure and an instantaneous pressure relaxation procedure.

The hyperbolic solver is detailed in [11]. Although perfectible, we use that solver here without modification. Improvements on that solver will be the subject of a forthcoming work.

The velocity relaxation procedure involves either the resolution of an ODE problem when the velocity relaxation has a finite rate, or an equilibrium procedure. That equilibrium procedure is the most useful one for the pre-cited applications. It will be recalled in this report but it does not pose any particular problem. That procedure consists in an exact analytical solution that is not very difficult to get.

The pressure relaxation procedure is much more difficult to solve. It is of paramount importance when dealing with interface problems (the pressure equality at the interface is the most difficult condition to fulfill). It is also of major importance for detonation, shocks and cavitation applications. The same type of procedure is necessary to solve in the context of the compressible model presented by Coquel et al. [4], and the same problematic is also present in the context of the various models and studies of Butler et al. [3], Kapila et al. [6] and Bdzil et al. [2].

That report is devoted to the assessment of this pressure relaxation procedure. We first present the two existing procedures of Saurel and Abgrall [11] which are two iterative procedures and also three other new algorithms among which two of them are quasi-direct procedures. Also, one of the new algorithm is more general in the sense that it allows the use of quite general EOS.

The paper is organized as follows. The 1-D model and its properties are first recalled. Then we detail the different relaxation procedures and at last we perform some representative numerical tests to compare those procedures.

\section{The 1-D two-phase model}

We are interested in a 1-D 2-phase flow problem with a gas phase ( $g$ index) and a liquid phase ( $l$ index), in which both mass and heat transfer are not considered. Since the two volume fractions verify

$$
\alpha_{g}+\alpha_{l}=1,
$$


the whole system to be solved reads

$$
\left\{\begin{array}{l}
\frac{\partial \alpha_{g}}{\partial t}+V_{I} \frac{\partial \alpha_{g}}{\partial x}=\mu\left(P_{g}-P_{l}\right), \\
\frac{\partial}{\partial t}\left(\alpha_{g} \rho_{g}\right)+\frac{\partial}{\partial x}\left(\alpha_{g} \rho_{g} u_{g}\right)=0, \\
\frac{\partial}{\partial t}\left(\alpha_{g} \rho_{g} u_{g}\right)+\frac{\partial}{\partial x}\left(\alpha_{g} \rho_{g} u_{g}^{2}+\alpha_{g} P_{g}\right)=P_{I} \frac{\partial \alpha_{g}}{\partial x}-\lambda\left(u_{g}-u_{l}\right), \\
\frac{\partial}{\partial t}\left(\alpha_{g} \rho_{g} E_{g}\right)+\frac{\partial}{\partial x}\left[u_{g}\left(\alpha_{g} \rho_{g} E_{g}+\alpha_{g} P_{g}\right)\right]=P_{I} V_{I} \frac{\partial \alpha_{g}}{\partial x}+\mu P_{I}\left(P_{g}-P_{l}\right)-\lambda V_{I}\left(u_{g}-u_{l}\right) \\
\frac{\partial}{\partial t}\left(\alpha_{l} \rho_{l}\right)+\frac{\partial}{\partial x}\left(\alpha_{l} \rho_{l} u_{l}\right)=0, \\
\frac{\partial}{\partial t}\left(\alpha_{l} \rho_{l} u_{l}\right)+\frac{\partial}{\partial x}\left(\alpha_{l} \rho_{l} u_{l}^{2}+\alpha_{l} P_{l}\right)=-P_{I} \frac{\partial \alpha_{g}}{\partial x}+\lambda\left(u_{g}-u_{l}\right), \\
\frac{\partial}{\partial t}\left(\alpha_{l} \rho_{l} E_{l}\right)+\frac{\partial}{\partial x}\left[u_{l}\left(\alpha_{l} \rho_{l} E_{l}+\alpha_{l} P_{l}\right)\right]=-P_{I} V_{I} \frac{\partial \alpha_{g}}{\partial x}-\mu P_{I}\left(P_{g}-P_{l}\right)+\lambda V_{I}\left(u_{g}-u_{l}\right)
\end{array}\right.
$$

where $V_{I}$ denotes the interface velocity, that we choose equal to the velocity of the center of mass. As it will be shown in the following, it also corresponds to the equilibrium velocity

$$
V_{I}=\frac{\alpha_{g} \rho_{g} u_{g}+\alpha_{l} \rho_{l} u_{l}}{\alpha_{g} \rho_{g}+\alpha_{l} \rho_{l}},
$$

$P_{I}$ is the interface pressure estimated equal to the mixture pressure

$$
P_{I}=\alpha_{g} P_{g}+\alpha_{l} P_{l}
$$

$u_{k}, \rho_{k}, P_{k}$ and $E_{k}(k=g, l)$, denote respectively the $k$-phase velocity, density, pressure and total energy. The $\lambda$ and $\mu$ functions are related to the relaxation effects in the velocity and pressure respectively. More details will be given in the following.

The total energy $E_{k}$ is related to the internal energy $e_{k}$ and the kinetic energy by the following classical relation

$$
E_{k}=e_{k}+\frac{1}{2} u_{k}^{2}
$$

The system is completed by two equations of state relying each pressure $P_{k}$ to the internal energy $e_{k}$ of the following form

$$
P_{k}=P_{k}\left(\rho_{k}, e_{k}\right) \text {. }
$$

The most classical EOS used for multiphase flows are given below.

1. Stiffened gas EOS:

$$
P_{k}=\left(\gamma_{k}-1\right) \rho_{k} e_{k}-\gamma_{k} P_{k, \infty}
$$

where $\gamma_{k}$ and $P_{k, \infty}$ are two non negative constant numbers. When $P_{k, \infty}=0$, we recover the classical perfect gas EOS and in that case $\gamma_{k}$ is the classical specific heat ratio: $\gamma_{k}=\left(C_{P}\right)_{k} /\left(C_{V}\right)_{k}$.

2. Van der Walls EOS:

$$
P_{k}=\frac{R_{k} T_{k}}{V_{k}-b_{k}}-\frac{C_{k}}{V_{k}^{2}},
$$

where $R_{k}$ is the mass constant of the $k$-phase, $T_{k}$ is the temperature, $V_{k}=1 / \rho_{k}$ is the specific volume, $b_{k}$ is the covolume, i.e. the volume occupied by the molecules and is supposed to be constant and positive, $C_{k}$ is also a positive contant. This EOS is often used when phase changes occur (boiling, cavitation or condensation). But it can be shown that this EOS does not fulfill the convexity requirement for an accurate solution with gas dynamic equations (Godunov at al. [5], Menikoff and Plohr [7]). In the context of the multiphase model representated by system (2), each phase possesses its own EOS, and such a mixture EOS like the van der Walls one is useless. We will not consider such type of EOS in this report.

\section{Mie Gruneisen EOS :}

$$
P_{k}=\left(\gamma_{k}\left(\rho_{k}\right)-1\right) \rho_{k} e_{k}-\gamma_{k}\left(\rho_{k}\right) P_{k, \infty}\left(\rho_{k}\right),
$$

it is in some sense a generalization of the previous equations of state and is generally used for solid, liquid as well as gas at very high pressure. The main difficulty consists in determining the functions of $\rho_{k}$, i.e. $\gamma_{k}$ and $P_{k, \infty}$. 


\subsection{Properties of the system}

We may rewrite system (2) in terms of physical variables

$$
W=\left(\alpha_{g}, \rho_{g}, u_{g}, P_{g}, \rho_{l}, u_{l}, P_{l}\right)^{T},
$$

by

$$
\frac{\partial W}{\partial t}+A(W) \frac{\partial W}{\partial x}=0
$$

where

$$
A(W)=\left(\begin{array}{ccccccc}
V_{I} & 0 & & 0 & 0 & 0 & 0 \\
\frac{\rho_{g}}{\alpha_{g}}\left(u_{g}-V_{I}\right) & u_{g} & \rho_{g} & 0 & 0 & 0 & 0 \\
\frac{P_{g}-P_{I}}{\alpha_{g} \rho_{g}} & 0 & u_{g} & 1 / \rho_{g} & 0 & 0 & 0 \\
\frac{\rho_{g} c_{g, I}^{2}}{\alpha_{g}}\left(u_{g}-V_{I}\right) & 0 & \rho_{g} c_{g}^{2} & u_{g} & 0 & 0 & 0 \\
-\frac{\rho_{l}}{\alpha_{l}}\left(u_{l}-V_{I}\right) & 0 & 0 & 0 & u_{l} & \rho_{l} & 0 \\
-\frac{P_{l}-P_{I}}{\alpha_{l} \rho_{l}} & 0 & 0 & 0 & 0 & u_{l} & 1 / \rho_{l} \\
-\frac{\rho_{l} c_{g, I}^{2}}{\alpha_{l}}\left(u_{l}-V_{I}\right) & 0 & 0 & 0 & 0 & \rho_{l} c_{l}^{2} & u_{l}
\end{array}\right),
$$

and

$$
c_{k}^{2}=\frac{\frac{P_{k}}{\rho_{k}^{2}}-\left.\frac{\partial e_{k}}{\partial \rho_{k}}\right|_{P_{k}}}{\left.\frac{\partial e_{k}}{\partial P_{k}}\right|_{\rho_{k}}}, c_{k, I}^{2}=\frac{\frac{P_{I}}{\rho_{k}^{2}}-\left.\frac{\partial e_{k}}{\partial \rho_{k}}\right|_{P_{k}}}{\left.\frac{\partial e_{k}}{\partial P_{k}}\right|_{\rho_{k}}},
$$

represent respectively the $k$-phase sound speed and the $k$-phase sound speed at the interface pressure condition. We may easily show that this matrix admits seven real and distinct eigenvalues which are $(k=g, l)$

$$
\left\{\begin{array}{l}
\lambda_{I}=V_{I} \\
\lambda_{k}^{+}=u_{k}+c_{k} \\
\lambda_{k}^{-}=u_{k}-c_{k} \\
\lambda_{k}^{0}=u_{k}
\end{array}\right.
$$

We can thus state that, except in the case where the eigenvalues are locally degenerated, that system is strictly hyperbolic and therefore diagonalizable.

\subsection{Numerical solution and pressure relaxation.}

The numerical solution of system (2) requires a hyperbolic solver and two relaxation procedures for the source terms resolution, provided that Strang splitting [12] is assumed to be valid.

We first recall the definition of the hyperbolic solver as given in Abgrall and Saurel [11]. 


\subsubsection{Hyperbolic solver}

The hyperbolic part of system (2) reads

$$
\left\{\begin{array}{l}
\frac{\partial \alpha_{g}}{\partial t}+V_{I} \frac{\partial \alpha_{g}}{\partial x}=0, \\
\frac{\partial}{\partial t}\left(\alpha_{g} \rho_{g}\right)+\frac{\partial}{\partial x}\left(\alpha_{g} \rho_{g} u_{g}\right)=0, \\
\frac{\partial}{\partial t}\left(\alpha_{g} \rho_{g} u_{g}\right)+\frac{\partial}{\partial x}\left(\alpha_{g} \rho_{g} u_{g}^{2}+\alpha_{g} P_{g}\right)=P_{I} \frac{\partial \alpha_{g}}{\partial x}, \\
\frac{\partial}{\partial t}\left(\alpha_{g} \rho_{g} E_{g}\right)+\frac{\partial}{\partial x}\left[u_{g}\left(\alpha_{g} \rho_{g} E_{g}+\alpha_{g} P_{g}\right)\right]=P_{I} V_{I} \frac{\partial \alpha_{g}}{\partial x} \\
\frac{\partial}{\partial t}\left(\alpha_{l} \rho_{l}\right)+\frac{\partial}{\partial x}\left(\alpha_{l} \rho_{l} u_{l}\right)=0, \\
\frac{\partial}{\partial t}\left(\alpha_{l} \rho_{l} u_{l}\right)+\frac{\partial}{\partial x}\left(\alpha_{l} \rho_{l} u_{l}^{2}+\alpha_{l} P_{l}\right)=-P_{I} \frac{\partial \alpha_{g}}{\partial x}, \\
\frac{\partial}{\partial t}\left(\alpha_{l} \rho_{l} E_{l}\right)+\frac{\partial}{\partial x}\left[u_{l}\left(\alpha_{l} \rho_{l} E_{l}+\alpha_{l} P_{l}\right)\right]=-P_{I} V_{I} \frac{\partial \alpha_{g}}{\partial x},
\end{array}\right.
$$

The hyperbolic solver is composed of a non conservative scheme to solve the first equation of that sytem which reads as (second order extension of the HLL solver, see [11], p. 443)

$$
\begin{aligned}
& \alpha_{i}^{n+1}=\alpha_{i}^{n} \\
& -\lambda\left[\frac{u_{i}^{n+\frac{1}{2}}\left(S_{i+\frac{1}{2}}^{+} \alpha_{i+\frac{1}{2},-}^{n+\frac{1}{2}}-S_{i+\frac{1}{2}}^{-} \alpha_{i+\frac{1}{2},+}^{n+\frac{1}{2}}\right)+S_{i+\frac{1}{2}}^{+} S_{i+\frac{1}{2}}^{-}\left(\alpha_{i+\frac{1}{2},+}^{n+\frac{1}{2}}-\alpha_{i+\frac{1}{2},-}^{n+\frac{1}{2}}\right)}{S_{i+\frac{1}{2}}^{+}-S_{i+\frac{1}{2}}^{-}}\right. \\
& \left.-\frac{u_{i}^{n+\frac{1}{2}}\left(S_{i-\frac{1}{2}}^{+} \alpha_{i-\frac{1}{2},-}^{n+\frac{1}{2}}-S_{i-\frac{1}{2}}^{-} \alpha_{i-\frac{1}{2},+}^{n+\frac{1}{2}}\right)+S_{i-\frac{1}{2}}^{+} S_{i-\frac{1}{2}}^{-}\left(\alpha_{i-\frac{1}{2},+}^{n+\frac{1}{2}}-\alpha_{i-\frac{1}{2},-}^{n+\frac{1}{2}}\right)}{S_{i-\frac{1}{2}}^{+}-S_{i-\frac{1}{2}}^{-}}\right],
\end{aligned}
$$

where $\lambda=\Delta t / \Delta x$, and a quasi-conservative scheme to solve the remaining 6 equations

$$
U_{i}^{n+1}=U_{i}^{n}-\lambda\left(F\left(U_{i+\frac{1}{2},-}^{n+\frac{1}{2}}, U_{i+\frac{1}{2},+}^{n+\frac{1}{2}}\right)-F\left(U_{i-\frac{1}{2},-}^{n}, U_{i-\frac{1}{2},+}^{n}\right)\right)+\Delta t H\left(U_{i}^{n+\frac{1}{2}}\right) \Delta
$$

where

$$
\Delta=\frac{1}{\Delta x}\left[\frac{S_{i+\frac{1}{2}}^{+} \alpha_{i+\frac{1}{2},-}^{n+\frac{1}{2}}-S_{i+\frac{1}{2}}^{-} \alpha_{i+\frac{1}{2},+}^{n+\frac{1}{2}}}{S_{i+\frac{1}{2}}^{+}-S_{i+\frac{1}{2}}^{-}}-\frac{S_{i-\frac{1}{2}}^{+} \alpha_{i-\frac{1}{2},-}^{n+\frac{1}{2}}-S_{i-\frac{1}{2}}^{-} \alpha_{i-\frac{1}{2},+}^{n+\frac{1}{2}}}{S_{i-\frac{1}{2}}^{+}-S_{i-\frac{1}{2}}^{-}}\right]
$$

The numerical fluxes $F\left(U_{i+\frac{1}{2},-}^{n+\frac{1}{2}}, U_{i+\frac{1}{2},+}^{n+\frac{1}{2}}\right)$ are given by the HLL approximate Riemann solver which reads

$$
F_{\mathrm{HLL}}=\frac{S^{+} F_{L}-S^{-} F_{R}+S^{+} S^{-}\left(U_{R}-U_{L}\right)}{S^{+}-S^{-}} .
$$

All details and notations are given in Abgrall and Saurel [11].

\subsubsection{Velocity relaxation}

In many situations, and in particular for interface problems solved by system (2), instantaneous velocity relaxation is necessary to satisfy the first interface condition corresponding to a normal velocities equality. The 
instantaneous velocity relaxation procedure consists in solving the following ODE system when $\lambda \rightarrow \infty$

$$
\begin{cases}\frac{\partial \alpha_{g}}{\partial t} & =0, \\ \frac{\partial \alpha_{g} \rho_{g}}{\partial t} & =0, \\ \frac{\partial \alpha_{g} \rho_{g} u_{g}}{\partial t} & =-\lambda\left(u_{g}-u_{l}\right), \\ \frac{\partial \alpha_{g} \rho_{g} E_{g}}{\partial t} & =-\lambda V_{I}\left(u_{g}-u_{l}\right), \\ \frac{\partial \alpha_{l} \rho_{l}}{\partial t} & =0, \\ \frac{\partial \alpha_{l} \rho_{l} u_{l}}{\partial t} & =+\lambda\left(u_{g}-u_{l}\right), \\ \frac{\partial \alpha_{l} \rho_{l} E_{l}}{\partial t} & =+\lambda V_{I}\left(u_{g}-u_{l}\right) .\end{cases}
$$

Solution of that ODE problem in the limit $\lambda \rightarrow \infty$ is detailed in Abgrall and Saurel [11]. Let " 0 " be the index symbol for the solution obtained after the hyperbolic solution step and "** be the index symbol for the solution gotten after the velocity relaxation procedure, then we get

$$
\left\{\begin{aligned}
\alpha_{g}^{*} & =\alpha_{g}^{0} \\
\rho_{g}^{*} & =\rho_{g}^{0} \\
\rho_{l}^{*} & =\rho_{l}^{0} \\
u_{l}^{*} & =u_{g}^{*}=V_{I}^{*}=V_{I}^{0}=\frac{\alpha_{g}^{0} \rho_{g}^{0} u_{g}^{0}+\alpha_{l}^{0} \rho_{l}^{0} u_{l}^{0}}{\alpha_{g}^{0} \rho_{g}^{0}+\alpha_{l}^{0} \rho_{l}^{0}} \\
e_{g}^{*} & =e_{g}^{0}+\frac{1}{2}\left(u_{g}^{*}-u_{g}^{0}\right)^{2} \\
e_{l}^{*} & =e_{l}^{0}+\frac{1}{2}\left(u_{l}^{*}-u_{l}^{0}\right)^{2}
\end{aligned}\right.
$$

\subsubsection{Instantaneous pressure relaxation}

When dealing with interface problems solved with system (2), the second interface condition that needs to be fulfilled is the pressure equality at the interface.

A way to satisfy that condition is to relax the pressures between phases towards equilibrium

$$
P_{g}=P_{l} .
$$

When dealing with compressible material mixtures, there are situations for which pressure equilibrium is reached so fast that the knowledge of the details of the pressure relaxation process is not usefull. Examples are shock propagation in solid alloys under strong shock waves, detonation in solid explosives etc. [10].

There is another important reason for which the relaxation procedure is important. The unconditional hyperbolicity of system (2) is a consequence of the compressibility of the phases. But there are a lot of situations for which the pressure differential between phases is negligible: almost all situations with liquid and gas mixtures at low velocity. The sound speed in the liquid phase being very fast compared to the convection velocity, most models assume pressure equilibrium between phases. When such an asssumption is made, the resulting model is no longer hyperbolic and numerical instabilities occur.

Our strategy is to consider each material as a compresible fluid to let the waves evolve during the hyperbolic solution, and then to make the pressures relax towards an equilibrium state. With such a strategy, numerical instabilities are removed. The aim of this report is to study the importance of this relaxation step, to improve its accuracy and computational efficiency.

The pressure relaxation procedure consists in solving the following ODE system in the limit $\mu \rightarrow \infty$

$$
\frac{\partial U}{\partial t}=\operatorname{PR}(U),
$$


where $U$ is the vector of conservative variables

$$
U=\left(\alpha_{g}, \alpha_{g} \rho_{g}, \alpha_{g} \rho_{g} u_{g}, \alpha_{g} \rho_{g} E_{g}, \alpha_{l} \rho_{l}, \alpha_{l} \rho_{l} u_{l}, \alpha_{l} \rho_{l} E_{l}\right)^{T},
$$

and PR is the pressure relaxation operator defined by

$$
\operatorname{PR}(U)=\left(\begin{array}{l}
\mu\left(P_{g}-P_{l}\right) \\
0 \\
0 \\
-\mu P_{I}\left(P_{g}-P_{l}\right) \\
0 \\
0 \\
\mu P_{I}\left(P_{g}-P_{l}\right)
\end{array}\right) .
$$

The $\mu$ variable represents an homogeneization parameter controlling the rate at which pressures tend towards equilibrium. Its physical meaning has been justified with considerations based on the second law of thermodynamics by Baer and Nunziato [1].

In the general case, its is a very complicated function of all flow variables and micro-structure of the mixture. With our approach, knowledge of this function is useless since pressure equilibrium is always assumed very fast compared to the all other time scales.

After solving the system (2) at time $t_{n}$ and velocity relaxation step if necessary, the pressure relaxation procedure consists in solving the following differential system

$$
\partial t U=\operatorname{PR}(U)
$$

i.e., during that process, the second, third, fifth and sixth components of the vector $U^{n}$ denoting the solution vector of the system (2) at time $t_{n}$, are constant.

Using the first equation, we may rewrite the energy equations as

$$
\left\{\begin{array}{l}
\frac{\partial}{\partial t}\left(\alpha_{g} \rho_{g} E_{g}\right)=-P_{I} \frac{\partial \alpha_{g}}{\partial t}, \\
\frac{\partial}{\partial t}\left(\alpha_{l} \rho_{l} E_{l}\right)=P_{I} \frac{\partial \alpha_{g}}{\partial t} .
\end{array}\right.
$$

Denoting by $T_{0}=t_{n}$ the initialization time of the pressure relaxation process and by $T_{*}$ the final time of the process (i.e. the pressure equilibrium final time) and integrating the equations in $\left[T_{0}, T_{*}\right]$, we get

$$
\left\{\begin{aligned}
\left(\alpha_{g} \rho_{g} E_{g}\right)^{*}-\left(\alpha_{g} \rho_{g} E_{g}\right)^{(0)} & =-\int_{T_{0}}^{T_{*}} P_{I} \frac{\partial \alpha_{g}}{\partial t} d t \\
\left(\alpha_{l} \rho_{l} E_{l}\right)^{*}-\left(\alpha_{l} \rho_{l} E_{l}\right)^{(0)} & =\int_{T_{0}}^{T_{*}} P_{I} \frac{\partial \alpha_{g}}{\partial t} d t
\end{aligned}\right.
$$

that is, by using the following change of variables

$$
\begin{gathered}
d \alpha_{g}=\frac{\partial \alpha_{g}}{\partial t} d t \\
\left\{\begin{array}{c}
\left(\alpha_{g} \rho_{g} E_{g}\right)^{*}-\left(\alpha_{g} \rho_{g} E_{g}\right)^{(0)}=-\int_{\alpha_{g}^{(0)}}^{\alpha_{g}^{*}} P_{I} d \alpha_{g}, \\
\left(\alpha_{l} \rho_{l} E_{l}\right)^{*}-\left(\alpha_{l} \rho_{l} E_{l}\right)^{(0)}=\int_{\alpha_{g}^{(0)}}^{\alpha_{g}^{*}} P_{I} d \alpha_{g},
\end{array}\right.
\end{gathered}
$$

where the zero index corresponds to the values taken at $t=T_{0}\left(=t_{n}\right)$, and the ${ }^{*}$ index denotes the values taken at the final relaxation time $t=T_{*}$. We may then proceed in two ways: either the pressure variation is high and in that case we may prefer to use an iterative numerical procedure to get the approximated solution where the integral in the right hand side will be approximated, or the pressure variation is low and in that case we may use a direct solution procedure after the integral has been approximated depending on the EOS of each fluid involved.

Let us first observe the case where the pressure variation is low. 


\subsubsection{A direct solution procedure for low pressure variation}

When dealing with fluids governed by the stiffened gas EOS and when pressure variations are low, a fast and direct relaxed state may be obtained with the following procedure. Let us recall that the stiffened gas EOS is a good approximation of the behaviour of most pure liquids and that its analytical formulation contains those of ideal gases. So most liquid-gas mixtures can be described by a stiffened gas EOS for each fluid.

Also, the limit of weak pressure variations is valid for most liquid-gas two-phase flows since their velocity (or more precisely their Mach number) is usually low.

Let $\varepsilon$ be a given arbitrary small positive number. If, for a given cell inside the domain of spatial discretization we assume the following approximation

$$
\int_{\alpha_{g}^{(0)}}^{\alpha_{g}^{*}} P_{I} d \alpha_{g} \simeq \frac{P_{I}^{(0)}+P_{I}^{*}}{2} \Delta \alpha_{g}^{*},
$$

where

$$
\Delta \alpha_{g}^{*}=\alpha_{g}^{*}-\alpha_{g}^{(0)}
$$

we get

$$
\left\{\begin{aligned}
\left(\alpha_{g} \rho_{g} E_{g}\right)^{*}-\left(\alpha_{g} \rho_{g} E_{g}\right)^{(0)} & =-\frac{P_{I}^{(0)}+P_{I}^{*}}{2} \Delta \alpha_{g}^{*} \\
\left(\alpha_{l} \rho_{l} E_{l}\right)^{*}-\left(\alpha_{l} \rho_{l} E_{l}\right)^{(0)} & =\frac{P_{I}^{(0)}+P_{I}^{*}}{2} \Delta \alpha_{g}^{*} .
\end{aligned}\right.
$$

Since

(28) also reads

$$
E_{k}=e_{k}+\frac{1}{2} u_{k}^{2} \text { and }\left(\alpha_{k} \rho_{k}\right)^{(0)}=\left(\alpha_{k} \rho_{k}\right)^{*}
$$

$$
\left\{\begin{aligned}
\left(\alpha_{g} \rho_{g}\right)^{(0)}\left(e_{g}^{*}-e_{g}^{(0)}\right) & =-\frac{P_{I}^{(0)}+P_{I}^{*}}{2} \Delta \alpha_{g}^{*} \\
\left(\alpha_{l} \rho_{l}\right)^{(0)}\left(e_{l}^{*}-e_{l}^{(0)}\right) & =\frac{P_{I}^{(0)}+P_{I}^{*}}{2} \Delta \alpha_{g}^{*}
\end{aligned}\right.
$$

If the equation of state is given by the stiffened gas law, we have

$$
e_{k}=\frac{P_{k}+\gamma_{k} P_{k, \infty}}{\left(\gamma_{k}-1\right) \rho_{k}} .
$$

Thus, by replacing that expression in (29), we get

$$
\left\{\begin{aligned}
\left(\alpha_{g} \rho_{g}\right)^{(0)}\left[\frac{\alpha_{g}^{(0)}\left[P_{g}^{(0)}+\gamma_{g} P_{g, \infty}\right]}{\left(\gamma_{g}-1\right)\left(\alpha_{g} \rho_{g}\right)^{(0)}}-\frac{\alpha_{g}^{*}\left[P_{g}^{*}+\gamma_{g} P_{g, \infty}\right]}{\left(\gamma_{g}-1\right)\left(\alpha_{g} \rho_{g}\right)^{(0)}}\right]=\frac{P_{I}^{(0)}+P_{g}^{*}}{2} \Delta \alpha_{g}^{*}, \\
\left(\alpha_{l} \rho_{l}\right)^{(0)}\left[\frac{\alpha_{l}^{*}\left[P_{l}^{*}+\gamma_{l} P_{l, \infty}\right]}{\left(\gamma_{l}-1\right)\left(\alpha_{l} \rho_{l}\right)^{(0)}}-\frac{\alpha_{l}^{(0)}\left[P_{l}^{(0)}+\gamma_{l} P_{l, \infty}\right]}{\left(\gamma_{l}-1\right)\left(\alpha_{l} \rho_{l}\right)^{(0)}}\right]=\frac{P_{I}^{(0)}+P_{l}^{*}}{2} \Delta \alpha_{g}^{*},
\end{aligned}\right.
$$

i.e.

$$
\left\{\begin{aligned}
& \alpha_{g}^{(0)}\left[P_{g}^{(0)}+\gamma_{g} P_{g, \infty}\right]-\alpha_{g}^{*}\left[P_{g}^{*}+\gamma_{g} P_{g, \infty}\right]=\left(\gamma_{g}-1\right) \frac{P_{I}^{(0)}+P_{g}^{*}}{2} \Delta \alpha_{g}^{*}, \\
& \alpha_{l}^{*}\left[P_{l}^{*}+\gamma_{l} P_{l, \infty}\right]-\alpha_{l}^{(0)}\left[P_{l}^{(0)}+\gamma_{l} P_{l, \infty}\right]=\left(\gamma_{l}-1\right) \frac{P_{I}^{(0)}+P_{l}^{*}}{2} \Delta \alpha_{g}^{*} .
\end{aligned}\right.
$$

From the two relations given above, we can deduce the following expressions for $P_{g}^{*}$ and $P_{l}^{*}$

$$
\left\{\begin{array}{c}
P_{g}^{*}=\frac{\alpha_{g}^{(0)} P_{g}^{(0)}-\left(\frac{\gamma_{g}-1}{2} P_{I}^{(0)}+\gamma_{g} P_{g, \infty}\right) \Delta \alpha_{g}^{*}}{\alpha_{g}^{(0)}+\frac{\gamma_{g}+1}{2} \Delta \alpha_{g}^{*}} \\
P_{l}^{*}=\frac{\alpha_{l}^{(0)} P_{l}^{(0)}+\left(\frac{\gamma_{l}-1}{2} P_{I}^{(0)}+\gamma_{l} P_{l, \infty}\right) \Delta \alpha_{g}^{*}}{\alpha_{l}^{(0)}-\frac{\gamma_{l}+1}{2} \Delta \alpha_{g}^{*}}
\end{array}\right.
$$


Since $P_{g}^{*}=P_{l}^{*}$, we get the following equation for $\Delta \alpha_{g}^{*}$

$$
A_{0}+A_{1} \Delta \alpha_{g}^{*}+A_{2}\left(\Delta \alpha_{g}^{*}\right)^{2}=0,
$$

with

$$
\left\{\begin{aligned}
A_{0} & =\alpha_{g}^{(0)} \alpha_{l}^{(0)} \Delta P^{(0)} \\
A_{1} & =\frac{\gamma_{l}+1}{2} \alpha_{g}^{(0)} P_{g}^{(0)}-\frac{\gamma_{g}+1}{2} \alpha_{l}^{(0)} P_{l}^{(0)} \\
& -\frac{\alpha_{l}^{(0)}\left(\gamma_{g}-1\right)+\alpha_{g}^{(0)}\left(\gamma_{l}-1\right)}{2} P_{I}^{(0)} \\
& -\left(\alpha_{l}^{(0)} \gamma_{g} P_{g, \infty}+\alpha_{g}^{(0)} \gamma_{l} P_{l, \infty}\right), \\
A_{2} & =\frac{\gamma_{g}-\gamma_{l}}{2} P_{I}^{(0)}+\frac{\gamma_{l}+1}{2} \gamma_{g} P_{g, \infty}-\frac{\gamma_{g}+1}{2} \gamma_{l} P_{l, \infty} .
\end{aligned}\right.
$$

Noticing that (32) can be rewritten as

$$
\left\{\begin{aligned}
\alpha_{g}^{(0)}\left(P_{g}^{(0)}-P_{g}^{*}\right) & =\left[\frac{\gamma_{g}-1}{2} P_{I}^{(0)}+\frac{\gamma_{g}+1}{2} P_{g}^{*}+\gamma_{g} P_{g, \infty}\right] \Delta \alpha_{g}^{*} \\
\alpha_{l}^{(0)}\left(P_{l}^{*}-P_{l}^{(0)}\right) & =\left[\frac{\gamma_{l}-1}{2} P_{I}^{(0)}+\frac{\gamma_{l}+1}{2} P_{l}^{*}+\gamma_{l} P_{l, \infty}\right] \Delta \alpha_{g}^{*}
\end{aligned}\right.
$$

we deduce that

$$
\begin{aligned}
\Delta P^{(0)}= & {\left[\frac{1}{\alpha_{g}^{(0)}}\left[\frac{\gamma_{g}-1}{2} P_{I}^{(0)}+\frac{\gamma_{g}+1}{2} P_{g}^{*}+\gamma_{g} P_{g, \infty}\right]\right.} \\
& \left.+\frac{1}{\alpha_{l}^{(0)}}\left[\frac{\gamma_{l}-1}{2} P_{I}^{(0)}+\frac{\gamma_{l}+1}{2} P_{l}^{*}+\gamma_{l} P_{l, \infty}\right]\right] \Delta \alpha_{g}^{*},
\end{aligned}
$$

and therefore

$$
\operatorname{sign}\left(\Delta \alpha_{g}^{*}\right)=\operatorname{sign}\left(\Delta P^{(0)}\right) .
$$

On the other hand, under the assumption that $\gamma_{l}>\gamma_{g}$, we have

$$
A_{2}<0 \text {, }
$$

therefore, the root product of the equation (34) has the same sign as $-\Delta P^{(0)}$. We conclude that the solution $\Delta \alpha_{g}^{*}$ is defined as follows

- if $\Delta P^{(0)}>0$, the two roots are of opposite signs and we therefore retain the positive root;

- if $\Delta P^{(0)}<0$, the two roots have the same sign and they are necessarily negative; we must retain the root $\Delta \alpha_{g}^{*}$ that satisfies

$$
\alpha_{g}^{*}=\alpha_{g}^{(0)}+\Delta \alpha_{g}^{*} \in[0,1],
$$

which ends the direct pressure relaxation process.

\subsubsection{Iterative relaxation procedures for high pressure variation}

When the pressure variation is high (as for shock tube problems for example) we may define some iterative procedure to get the approximate relaxed pressure $P^{*}$. We define hereafter the two existing iterative procedures first proposed in Abgrall and Saurel [11] and propose two other new procedures. The first of the new procedures uses the ideas of the existing procedures but controls the variation of the pressures (monotonicity) at each iteration and gives a systematic guess of the volume fraction as a function of that variation. The second new procedure is devoted to more general EOS and seems particularly well suited for problems with strong shocks and general materials. 
1. Procedure 1: in that procedure, we want to get a variation $\Delta \alpha_{g}^{(k)}=\alpha_{g}^{(k)}-\alpha_{g}^{(k-1)}$, at each iteration $k$, such that

$$
\left\{\begin{array}{l}
\left|\Delta P^{(k+1)}\right|<\left|\Delta P^{(k)}\right| \\
\operatorname{sign}\left(\Delta P^{(k+1)}\right)=\operatorname{sign}\left(\Delta P^{(k)}\right) .
\end{array}\right.
$$

To do this, we first give four positive parameters which will be used later on

$$
\varepsilon_{\alpha}, \varepsilon_{P}, \varepsilon_{0}, D_{\alpha}
$$

In that approach, the system (27) is replaced at each iteration $k$ by:

$$
\left\{\begin{array}{l}
\left(\alpha_{g} \rho_{g} E_{g}\right)^{(k+1)}-\left(\alpha_{g} \rho_{g} E_{g}\right)^{(0)}=-\int_{\alpha_{g}^{(0)}}^{\alpha_{g}^{(k+1)}} P_{I} d \alpha_{g}, \\
\left(\alpha_{l} \rho_{l} E_{l}\right)^{(k+1)}-\left(\alpha_{l} \rho_{l} E_{l}\right)^{(0)}=\int_{\alpha_{g}^{(0)}}^{\alpha_{g}^{(k+1)}} P_{I} d \alpha_{g},
\end{array}\right.
$$

and we opt for the following approximation of the pressure integral

$$
\int_{\alpha_{g}^{(0)}}^{\alpha_{g}^{(k+1)}} P_{I} d \alpha_{g} \simeq \sum_{j=0}^{j=k} P_{I}^{(j)} \Delta \alpha_{g}^{(j+1)}
$$

By using the following notations

$$
\left\{\begin{aligned}
\Delta_{+}^{(j)} & =P_{I}^{(j)} \Delta \alpha_{g}^{(j+1)} \\
\Sigma_{\alpha}^{(j)} & =\sum_{i=0}^{i=j} \Delta_{+}^{(i)} \\
& j=1, \ldots, k+1
\end{aligned}\right.
$$

we are looking for determining a good variation $\Delta \alpha_{g}^{(k+1)}$ such that, after the computation of the following energy equalities

$$
\left\{\begin{array}{l}
\left(\alpha_{g} \rho_{g} E_{g}\right)^{(k+1)}=\left(\alpha_{g} \rho_{g} E_{g}\right)^{(0)}-\Sigma_{\alpha}^{(k+1)} \\
\left(\alpha_{l} \rho_{l} E_{l}\right)^{(k+1)}=\left(\alpha_{l} \rho_{l} E_{l}\right)^{(0)}+\Sigma_{\alpha}^{(k+1)}
\end{array}\right.
$$

we get a pressure variation $\Delta P^{(k+1)}$ which satisfies

$$
\left|\Delta P^{(k+1)}\right|<\left|\Delta P^{(k)}\right|
$$

and the process will stop as soon as

$$
\left|\Delta P^{(k+1)}\right| / P_{I}^{(k+1)} \leq \varepsilon_{P}
$$

where $\varepsilon_{P}$ is a positive parameter given in ]0,1[, for which we consider the approximate equilibrium state to be attained.

Initially, we know the following quantities

$$
\alpha_{m}^{(0)}, \rho_{m}^{(0)}, u_{m}^{(0)}, e_{m}^{(0)}, m=g, l
$$

which correspond to the physical variables of the two phases computed in the prediction step at time $t_{n+1}$ by solving the system of 7 equations without taking into account the terms related to the pressure relaxation in the right hand sides. The computation is done in each internal cell for which we have

$$
\left\{\begin{array}{l}
\min \left(\alpha_{g}^{(0)}, \alpha_{l}^{(0)}\right)>\varepsilon_{\alpha} \\
\left|\Delta P^{(0)}\right| / P_{I}^{(0)}>\varepsilon_{P}
\end{array}\right.
$$

where $\varepsilon_{\alpha}$ and $\varepsilon_{P}$ are some given parameters in ]0,1[ below which relaxation is not performed. 
We know that the following conservative variables $\left(\alpha_{m} \rho_{m}\right)$ and $\left(\alpha_{m} \rho_{m} u_{m}\right)$ for $m=g, l$, remain constant during the relaxation process. We therefore deduce in particular that the velocities also remain contant. At the very first initialization $(k=1)$, we define a variation $\Delta \alpha_{g}^{(1)}$ by

$$
\Delta \alpha_{g}^{(1)}=\varepsilon_{0} \min \left(\alpha_{g}^{(0)}, \alpha_{l}^{(0)}\right)
$$

where $\left.\varepsilon_{0} \in\right] 0,1[$.

For a given iteration $k$, we thus know how computing the pressures $P_{g}^{(k+1)}, P_{l}^{(k+1)}$ and $P_{I}^{(k+1)}$ from (42). As a matter of fact, from (42), we can compute the internal energy for each phase

$$
\left\{\begin{array}{l}
e_{g}^{(k+1)}=\left(\alpha_{g} \rho_{g} E_{g}\right)^{(k+1)} /\left(\alpha_{g} \rho_{g}\right)^{(0)}-\frac{1}{2} u_{g}^{2} \\
e_{l}^{(k+1)}=\left(\alpha_{l} \rho_{l} E_{l}\right)^{(k+1)} /\left(\alpha_{l} \rho_{l}\right)^{(0)}-\frac{1}{2} u_{l}^{2}
\end{array}\right.
$$

and the densities are given by

$$
\left\{\begin{array}{l}
\rho_{g}^{(k+1)}=\left(\alpha_{g} \rho_{g}\right)^{(0)} / \alpha_{g}^{(k+1)}, \\
\rho_{l}^{(k+1)}=\left(\alpha_{l} \rho_{l}\right)^{(0)} /\left(1-\alpha_{g}^{(k+1)}\right),
\end{array}\right.
$$

with

$$
\alpha_{g}^{(k+1)}=\alpha_{g}^{(k)}+\Delta \alpha_{g}^{(k+1)} .
$$

From those quantities, we can indeed compute the corresponding pressures.

If (44) is satisfied, we stop the relaxation process for that cell and we thus have to correct (update) the following conservative variables $\alpha_{g},\left(\alpha_{g} \rho_{g} E_{g}\right)$ and $\left(\alpha_{l} \rho_{l} E_{l}\right)$

$$
\begin{cases}\alpha_{g} & =\alpha_{g}^{(k+1)} \\ \left(\alpha_{g} \rho_{g} E_{g}\right) & =\left(\alpha_{g} \rho_{g} E_{g}\right)^{(k+1)} \\ \left(\alpha_{l} \rho_{l} E_{l}\right) & =\left(\alpha_{l} \rho_{l} E_{l}\right)^{(k+1)}\end{cases}
$$

and we may inspect (if any) the next cell. Otherwise, two instances may occur

(a) $\Delta P^{(k+1)} \Delta P^{(k)}<0$ : i.e. the pressure variations are of opposite sign. In that case, we define a new guess value for $\Delta \alpha_{g}^{(k+1)}$ by setting

$$
\Delta \alpha_{g}^{(k+1)}=\Delta \alpha_{g}^{(k+1)} / D_{\alpha}
$$

where $D_{\alpha}$ is a real parameter greater than 1 , and we take the standard value 2 . We then restart all the process from that new guess value $\Delta \alpha_{g}^{(k+1)}$, i.e. we recompute all the quantities given by (41), (42), (47) and (48), from which we may deduce the new pressure values and retest them.

(b) $\Delta P^{(k+1)} \Delta P^{(k)} \geq 0$ : i.e. the pressure variations have the same sign. In that case, we first check if

$$
\left|\Delta P^{(k+1)}\right|<\left|\Delta P^{(k)}\right|
$$

if that test is true, we go to the next iteration, otherwise, we redo the iteration by defining a new guess value for the volumic fraction variation $\Delta \alpha_{g}^{(k+1)}$ by setting

$$
\Delta \alpha_{g}^{(k+1)}=-\Delta \alpha_{g}^{(k+1)} / D_{\alpha}
$$

That process guaranties a monotone variation of the pressure until the equilibrium state is reached. Note that this procedure do not use any explicit form of the EOS and so is valid for real materials. 
2. Procedure 2: In that procedure, we also try to define a variation guess $\Delta \alpha_{g}^{(k+1)}$, at each iteration $k$, except that the total energy conservative variables are computed from the following approximation of the pressure integral

$$
\int_{\alpha_{g}^{(0)}}^{\alpha_{g}^{(k+1)}} P_{I} d \alpha_{g} \simeq \frac{P_{I}^{(0)}+P_{I}^{(k+1)}}{2} \Delta \alpha_{g}^{(k+1)} .
$$

where that choice is motivated by the fact that we are looking for a $P_{I}^{(k+1)}$ which is as close as possible as the "true" relaxed pressure $P^{*}$. It is therefore, in some sense, a variant version of the direct computation procedure given in the previous section.

We suppose that that approximated equilibrium state is reached as soon as the following relative pressure variation

$$
\delta_{3} P^{(k+1)} \stackrel{\text { def }}{=} \frac{\left(P_{I}^{(k+1)}-P_{I}^{(k)}\right)}{P_{I}^{(0)}}
$$

is sufficiently small.

Let us then first give the following non negative parameters

$$
\varepsilon_{\alpha}, \varepsilon_{P}, \varepsilon_{0}, D_{\alpha}, \varepsilon_{\delta P}
$$

where $\left.\varepsilon_{\alpha} \in\right] 0,1\left[\right.$ and $\left.\varepsilon_{P} \in\right] 0,1[$ are used as in the previous procedure (Procedure 1$)$ in $\left.(45), \varepsilon_{0} \in\right] 0,1[$ is also used here to define the initial volumique fraction variation as given by in (46), $D_{\alpha}$ is also used to redefine, for a given iteration, a new variation guess $\Delta \alpha_{g}^{(k+1)}$ if the one initially given is not satisfactory and $\left.\varepsilon_{\delta P} \in\right] 0,1[$ is used as a threshold for the relative pressure variation (53), for defining the final approximated equilibrium pressure of $P^{*}$.

At the very first iteration, we start with the following initialization

$$
\left\{\begin{array}{l}
\alpha_{g}^{(1)}=\alpha_{g}^{(0)}, \\
P_{I}^{(1)}=P_{I}^{(0)} \\
\Delta \alpha_{g}^{(1)}=\varepsilon_{0} \min \left(\alpha_{g}^{(0)}, \alpha_{l}^{(0)}\right), \\
\delta P^{(0)}=C_{\delta},
\end{array}\right.
$$

where $C_{\delta}$ is a sixth non negative parameter arbitrarily large.

For a given iteration $k,(k \geq 1)$, we first compute the internal energies for each phase

$$
\left\{\begin{array}{l}
e_{g}^{(k)}=e_{g}^{(0)}-\frac{P_{I}^{(0)}+P_{I}^{(k)}}{2} \frac{\left(\alpha_{g}^{(k)}-\alpha_{g}^{(0)}\right)}{\left(\alpha_{g} \rho_{g}\right)^{(0)}}, \\
e_{l}^{(k)}=e_{l}^{(0)}+\frac{P_{I}^{(0)}+P_{I}^{(k)}}{2} \frac{\left(\alpha_{g}^{(k)}-\alpha_{g}^{(0)}\right)}{\left(\alpha_{g} \rho_{g}\right)^{(0)}}
\end{array}\right.
$$

and also the corresponding densities

$$
\left\{\begin{array}{l}
\rho_{g}^{(k)}=\left(\alpha_{g} \rho_{g}\right)^{(0)} / \alpha_{g}^{(k)} \\
\rho_{l}^{(k)}=\left(\alpha_{l} \rho_{l}\right)^{(0)} / \alpha_{l}^{(k)}
\end{array}\right.
$$

from which we may deduce the pressure values of $P_{g}^{(k)}$ and $P_{l}^{(k)}$.

We then define the following quantities

$$
\begin{cases}\delta P^{(k)} & \stackrel{\text { def }}{=} \Delta P^{(k)} / P_{I}^{(0)} \\ \pi_{\delta_{P}}^{(k)} & \stackrel{\text { def }}{=} \delta P^{(k)} \delta P^{(k-1)}\end{cases}
$$

and we do the following tests 
(a) If

$$
\left|\delta P^{(k)}\right| \leq \varepsilon_{P}
$$

and if

$$
\left|\delta_{3} P^{(k)}\right| \leq \varepsilon_{P},
$$

then we may state that $P_{I}^{(k)}$ is a good approximation for the relaxed pressure $P^{*}$. In that case, we update the conservative variables as follows

$$
\begin{cases}\alpha_{g} & =\alpha_{g}^{(k)}, \\ \left(\alpha_{g} \rho_{g} E_{g}\right) & =\left(\alpha_{g} \rho_{g}\right)^{(0)}\left(e_{g}^{(k)}+\frac{1}{2} u_{g}^{2}\right), \\ \left(\alpha_{l} \rho_{l} E_{l}\right) & =\left(\alpha_{l} \rho_{l}\right)^{(0)}\left(e_{l}^{(k)}+\frac{1}{2} u_{l}^{2}\right),\end{cases}
$$

and we can go to the next cell if any.

(b) Otherwise, one of the previous tests (58) or (59) is not satisfied and we proceed as follows

i. if the first test (58) is not satisfied (the relative pressure variation is supposed to be not negligible), and if the variation product $\pi_{\delta_{P}}^{(k)}$ satisfies

$$
\pi_{\delta_{P}}^{(k+1)}<0
$$

then we go to the next iteration after setting

$$
\left\{\begin{array}{c}
\Delta \alpha_{g}^{(k+1)}=-\Delta \alpha_{g}^{(k)} / D_{\alpha}, \\
\alpha_{g}^{(k+1)}=\alpha_{g}^{(k)}+\Delta \alpha_{g}^{(k+1)} ;
\end{array}\right.
$$

ii. if the first test (58) is not satisfied but if (61) is satisfied then

- either

$$
\left|\delta P^{(k)}\right| \leq\left|\delta P^{(k-1)}\right|
$$

and in that case we go to the next iteration by first setting

$$
\begin{cases}\Delta \alpha_{g}^{(k+1)} & =\Delta \alpha_{g}^{(k)}, \\ \alpha_{g}^{(k+1)} & =\alpha_{g}^{(k)}+\Delta \alpha_{g}^{(k+1)} .\end{cases}
$$

- or

$$
\left|\delta P^{(k)}\right|>\left|\delta P^{(k-1)}\right|
$$

and in that case, we go to the next iteration with the following setting

$$
\begin{cases}\Delta \alpha_{g}^{(k+1)} & =-\Delta \alpha_{g}^{(k)} \\ \alpha_{g}^{(k+1)} & =\alpha_{g}^{(k)}+\Delta \alpha_{g}^{(k+1)} .\end{cases}
$$

iii. If the first test (58) is satisfied but if the second test (59) is not, the we go to the next iteration with the following setting

$$
\begin{cases}\Delta \alpha_{g}^{(k+1)} & =\Delta \alpha_{g}^{(0)} \\ \alpha_{g}^{(k+1)} & =\alpha_{g}^{(k)}\end{cases}
$$

In that present procedure (Procedure 2), we do not control the monotonicity of the pressure variation at each iteration, but it is again valid for real material EOS.

3. Procedure 3: In this new procedure, the ingredients come from a deeper insight of how to define an iterative process to reach the equilibrium state. We first start by showing how we may define that process. 
We recall that we initially want to solve the system given by (25). If we integrate those equations in the time interval $\left[T_{k}, T_{k+1}\right]$, we get:

$$
\left\{\begin{aligned}
\int_{T_{k}}^{T_{k+1}} \frac{\partial}{\partial t}\left(\alpha_{g} \rho_{g} E_{g}\right) d t & =\left(\alpha_{g} \rho_{g} E_{g}\right)^{(k+1)}-\left(\alpha_{g} \rho_{g} E_{g}\right)^{(k)} \\
& =-\int_{T_{k}}^{T_{k+1}} P_{I} \frac{\partial \alpha_{g}}{\partial t} d t=-\int_{\alpha_{g}^{(k)}}^{\alpha_{g}^{(k+1)}} P_{I} d \alpha_{g}, \\
\int_{T_{k}}^{T_{k+1}} \frac{\partial}{\partial t}\left(\alpha_{l} \rho_{l} E_{l}\right) d t & =\left(\alpha_{l} \rho_{l} E_{l}\right)^{(k+1)}-\left(\alpha_{l} \rho_{l} E_{l}\right)^{(k)} \\
& =\int_{T_{k}}^{T_{k+1}} P_{I} \frac{\partial \alpha_{g}}{\partial t} d t=\int_{\alpha_{g}^{(k)}}^{\alpha_{g}^{(k+1)}} P_{I} d \alpha_{g} .
\end{aligned}\right.
$$

As $\left(\alpha_{j} \rho_{j}\right)$ and $u_{j}, j=g, l$ remain constant during the pressure relaxation processs, and since

$$
E_{j}=e_{j}+\frac{1}{2} u_{j}^{2},
$$

we then get

$$
\left\{\begin{aligned}
\left(\alpha_{g} \rho_{g}\right)^{(k)}\left[e_{g}^{(k+1)}-e_{g}^{(k)}\right] & =-\int_{\alpha_{g}^{(k)}}^{\alpha_{g}^{(k+1)}} P_{I} d \alpha_{g} \\
\left(\alpha_{l} \rho_{l}\right)^{(k)}\left[e_{l}^{(k+1)}-e_{l}^{(k)}\right] & =\int_{\alpha_{g}^{(k)}}^{\alpha_{g}^{(k+1)}} P_{I} d \alpha_{g}
\end{aligned}\right.
$$

Therefore, if choosing the following approximation

$$
\int_{\alpha_{g}^{(k)}}^{\alpha_{g}^{(k+1)}} P_{I} d \alpha_{g} \simeq \frac{P_{I}^{(k)}+P_{I}^{(k+1)}}{2} \Delta \alpha_{g}^{(k+1)},
$$

with

$$
\Delta \alpha_{g}^{(k+1)} \stackrel{\text { def }}{=} \alpha_{g}^{(k+1)}-\alpha_{g}^{(k)}
$$

we get

$$
\left\{\begin{aligned}
\left(\alpha_{g} \rho_{g}\right)^{(k)}\left[e_{g}^{(k+1)}-e_{g}^{(k)}\right] & =-\frac{P_{I}^{(k)}+P_{I}^{(k+1)}}{2} \Delta \alpha_{g}^{(k+1)} \\
\left(\alpha_{l} \rho_{l}\right)^{(k)}\left[e_{l}^{(k+1)}-e_{l}^{(k)}\right] & =\frac{P_{I}^{(k)}+P_{I}^{(k+1)}}{2} \Delta \alpha_{g}^{(k+1)} .
\end{aligned}\right.
$$

But, from the stiffened gas EOS and since $\left(\alpha_{j} \rho_{j}\right)$ is constant $(j=g, l)$, we may write, for $m=k, k+1$

$$
e_{j}^{(m)}=\frac{P_{j}^{(m)}+\gamma_{j} P_{j, \infty}}{\left(\gamma_{j}-1\right) \rho_{j}^{(m)}}=\frac{\alpha_{j}^{(m)}\left(P_{j}^{(m)}+\gamma_{j} P_{j, \infty}\right)}{\left(\gamma_{j}-1\right)\left(\alpha_{j} \rho_{j}\right)^{(m)}}
$$

i.e.

$$
\left\{\begin{aligned}
\left(\alpha_{g} \rho_{g}\right)^{(k)} \quad & {\left[\frac{\alpha_{g}^{(k+1)}\left(P_{g}^{(k+1)}+\gamma_{g} P_{g, \infty}\right)-\alpha_{g}^{(k)}\left(P_{g}^{(k)}-\gamma_{g} P_{g, \infty}\right)}{\left(\gamma_{g}-1\right)\left(\alpha_{g} \rho_{g}\right)^{(k)}}\right]=} \\
& -\frac{P_{I}^{(k)}+P_{I}^{(k+1)}}{2} \Delta \alpha_{g}^{(k+1)} \\
\left(\alpha_{l} \rho_{l}\right)^{(k)} \quad & {\left[\frac{\alpha_{l}^{(k+1)}\left(P_{l}^{(k+1)}+\gamma_{l} P_{l, \infty}\right)-\alpha_{l}^{(k)}\left(P_{l}^{(k)}-\gamma_{l} P_{l, \infty}\right)}{\left(\gamma_{l}-1\right)\left(\alpha_{l} \rho_{l}\right)^{(k)}}\right]=} \\
& \frac{P_{I}^{(k)}+P_{I}^{(k+1)}}{2} \Delta \alpha_{g}^{(k+1)}
\end{aligned}\right.
$$

that is

$$
\begin{cases}\alpha_{g}^{(k+1)} P_{g}^{(k+1)} & =\alpha_{g} P_{g}^{(k)}-\left[\frac{\gamma_{g}-1}{2}\left(P_{I}^{(k)}+P_{I}^{(k+1)}\right)+\gamma_{g} P_{g, \infty}\right] \Delta \alpha_{g}^{(k+1)} \\ \alpha_{l}^{(k+1)} P_{l}^{(k+1)} & =\alpha_{l} P_{l}^{(k)}+\left[\frac{\gamma_{l}-1}{2}\left(P_{I}^{(k)}+P_{I}^{(k+1)}\right)+\gamma_{l} P_{l, \infty}\right] \Delta \alpha_{g}^{(k+1)}\end{cases}
$$


By summing the two above equations, we finally get

$$
P_{I}^{(k+1)}=\frac{1+\frac{\gamma_{l}-\gamma_{g}}{2} \Delta \alpha_{g}^{(k+1)}}{1-\frac{\gamma_{l}-\gamma_{g}}{2} \Delta \alpha_{g}^{(k+1)}} P_{I}^{(k)}+\frac{\gamma_{l} P_{l, \infty}-\gamma_{g} P_{g, \infty}}{1-\frac{\gamma_{l}-\gamma_{g}}{2} \Delta \alpha_{g}^{(k+1)}} \Delta \alpha_{g}^{(k+1)} .
$$

Therefore, assuming $\Delta \alpha_{g}^{(k+1)}$ is defined, we may deduce the value of $P_{I}^{(k+1)}$ and therefore also the values of the internal energies for each phase

$$
\left\{\begin{array}{rl}
e_{g}^{(k+1)} & =e_{g}^{(k)}-\frac{P_{I}^{(k)}+P_{I}^{(k+1)}}{2} \frac{\Delta \alpha_{g}^{(k+1)}{ }^{(k)}}{\left(\alpha_{g} \rho_{g}\right)}, \\
e_{l}^{(k+1)} & =e_{l}^{(k)}+\frac{P_{I}^{(k)}+P_{I}^{(k+1)}}{2} \frac{\Delta \alpha_{g}^{(k+1)}{ }^{(k)}}{\left(\alpha_{l} \rho_{l}\right)}
\end{array} .\right.
$$

We can also get the densities since

$$
\left\{\begin{array}{l}
\rho_{g}^{(k+1)}=\frac{\left(\alpha_{g} \rho_{g}\right)^{(k)}}{\alpha_{g}^{(k+1)}}=\frac{\left(\alpha_{g} \rho_{g}\right)^{(0)}}{\alpha_{g}^{(k+1)}} \\
\rho_{l}^{(k+1)}=\frac{\left(\alpha_{l} \rho_{l}\right)^{(k)}}{\alpha_{l}^{(k+1)}}=\frac{\left(\alpha_{l} \rho_{l}\right)^{(0)}}{\alpha_{l}^{(k+1)}}
\end{array}\right.
$$

We can now get the pressure values of each phase

$$
\left\{\begin{array}{l}
P_{g}^{(k+1)}=\left(\gamma_{g}-1\right) \rho_{g}^{(k+1)} e_{g}^{(k+1)}-\gamma_{g} P_{g, \infty}, \\
P_{l}^{(k+1)}=\left(\gamma_{l}-1\right) \rho_{l}^{(k+1)} e_{l}^{(k+1)}-\gamma_{l} P_{l, \infty}
\end{array}\right.
$$

Therefore, if we know how to define a good variation $\Delta \alpha_{g}^{(k+1)}$, at each $k$ iteration, we only have to let us give the test parameter $\left.\varepsilon_{P} \in\right] 0,1[$ and to stop the process as soon as the relative pressure variation satisfies

$$
\frac{\left|P_{g}^{(k+1)}-P_{l}^{(k+1)}\right|}{P_{I}^{(k+1)}} \leq \varepsilon_{P} .
$$

A judicious definition of the iterative variation $\Delta \alpha_{g}^{(k+1)}$ is therefore the key point of that procedure.

We know that

$$
\left\{\begin{array}{l}
\alpha_{g}^{(k+1)}=\alpha_{g}^{(k)}+\Delta \alpha_{g}^{(k+1)} \\
\alpha_{l}^{(k+1)}=\alpha_{l}^{(k)}-\Delta \alpha_{g}^{(k+1)}
\end{array}\right.
$$

therefore, replacing those expressions in equations (73) leads to

$$
\left\{\begin{aligned}
P_{g}^{(k+1)}-P_{g}^{(k)} & =-\frac{1}{\alpha_{g}^{(k)}}\left[\frac{\gamma_{g}-1}{2}\left(P_{I}^{(k)}+P_{I}^{(k+1)}\right)+P_{g}^{(k+1)}+\gamma_{g} P_{g, \infty}\right] \Delta \alpha_{g}^{(k+1)}, \\
P_{l}^{(k+1)}-P_{l}^{(k)} & =\frac{1}{\alpha_{l}^{(k)}}\left[\frac{\gamma_{l}-1}{2}\left(P_{I}^{(k)}+P_{I}^{(k+1)}\right)+P_{l}^{(k+1)}+\gamma_{l} P_{l, \infty}\right] \Delta \alpha_{g}^{(k+1)} .
\end{aligned}\right.
$$

We set for $m=k, k+1$

$$
\Delta P^{(m)} \stackrel{\text { def }}{=} P_{g}^{(m)}-P_{l}^{(m)}
$$

and we get, after substracting the previous equations given above

$$
\begin{aligned}
\Delta P^{(k+1)}= & \Delta P^{(k)} \\
- & \left\{\frac{1}{\alpha_{g}^{(k)}}\left[\frac{\gamma_{g}-1}{2}\left(P_{I}^{(k)}+P_{I}^{(k+1)}\right)+P_{g}^{(k+1)}+\gamma_{g} P_{g, \infty}\right]\right. \\
& \left.+\frac{1}{\alpha_{l}^{(k)}}\left[\frac{\gamma_{l}-1}{2}\left(P_{I}^{(k)}+P_{I}^{(k+1)}\right)+P_{l}^{(k+1)}+\gamma_{l} P_{l, \infty}\right]\right\} \Delta \alpha_{g}^{(k+1)} .
\end{aligned}
$$


We deduce from that expression that to enforce

$$
\left|\Delta P^{(k+1)}\right|<\left|\Delta P^{(k)}\right|,
$$

we must necessarily have

$$
\operatorname{sign}\left(\Delta \alpha_{g}^{(k+1)}\right)=\operatorname{sign}\left(\Delta P^{(k)}\right),
$$

therefore

$$
\exists \mu>0, \Delta \alpha_{g}^{(k+1)}=\mu \Delta P^{(k)} .
$$

If such a $\mu$ exists, we must ensure that

$$
\alpha_{g}^{(k+1)}=\alpha_{g}^{(k)}+\mu \Delta P^{(k)} \in[0,1],
$$

which is equivalent to write that

$$
\begin{cases}0<\mu \leq \frac{\alpha_{l}^{(k)}}{\Delta P^{(k)}} & \text { if } \Delta P^{(k)}>0, \\ 0<\mu \leq-\frac{\alpha_{g}^{(k)}}{\Delta P^{(k)}} & \text { if } \Delta P^{(k)}<0 .\end{cases}
$$

So, it is sufficient to set

$$
\mu=\mu_{0} \frac{\min \left(\alpha_{g}^{(k)}, \alpha_{l}^{(k)}\right)}{\left|\Delta P^{(k)}\right|},
$$

where $\mu_{0}$ is a constant, $\left.\mu_{0} \in\right] 0,1[$. We may finally give the following expression

$$
\left.\Delta \alpha_{g}^{(k+1)}=\mu_{0} \operatorname{sign}\left(\Delta P^{(k)}\right) \min \left(\alpha_{g}^{(k)}, \alpha_{l}^{(k)}\right), \mu_{0} \in\right] 0,1[.
$$

It therefore remains to give a good definition of $\mu_{0}$. Returning to the definition of the interface pressure $P_{I}^{(k+1)}$ given by (74) and assuming that

$$
\Delta \alpha_{g}^{(k+1)}<\frac{2}{\gamma_{l}-\gamma_{g}},
$$

we must end up with an admissible value of that interfacial pressure, that has to be non negative. That leads to the two following cases

(a) if $\Delta P^{(k)}>0$ then, since $\Delta \alpha_{g}^{(k+1)}$ has the same sign and since (88) is satisfied, the definition of $P_{I}^{(k+1)}$ given by (74) leads indeed to a positive value;

(b) if $\Delta P^{(k)}<0$ then, positiveness of the interface pressure is achieved under the following condition

$$
\Delta \alpha_{g}^{(k+1)}>-\frac{P_{I}^{(k)}}{\frac{\gamma_{l}-\gamma_{g}}{2} P_{I}^{(k)}+\gamma_{l} P_{l, \infty}-\gamma_{g} P_{g, \infty}} .
$$

We can now state the final definition of the volume fraction variation $\Delta \alpha_{g}^{(k+1)}$ :

given the pressure values $P_{I}^{(k)}, P_{g}^{(k)}$ and $P_{l}^{(k)}$ and the following initial guess definition of $\Delta \alpha_{g}^{(k+1)}$ :

$$
\Delta \alpha_{g}^{(k+1)} \stackrel{\text { def }}{=} \min \left(\mu_{0} \operatorname{sign}\left(\Delta P^{(k)}\right) \min \left(\alpha_{g}^{(k)}, \alpha_{l}^{(k)}\right), \frac{2}{\gamma_{l}-\gamma_{g}}\right)
$$

that definition remains if $\Delta P^{(k)}$ is positive, otherwise, it is modified by the following strategy

$$
\text { While } \quad \begin{aligned}
\Delta \alpha_{g}^{(k+1)} & \leq C^{(k)} \text { do } \\
\Delta \alpha_{g}^{(k+1)} & \longleftarrow \Delta \alpha_{g}^{(k+1)} / 2
\end{aligned}
$$


where

$$
C^{(k)}=-\frac{P_{I}^{(k)}}{\frac{\gamma_{l}-\gamma_{g}}{2} P_{I}^{(k)}+\gamma_{l} P_{l, \infty}-\gamma_{g} P_{g, \infty}} .
$$

That ends the definition of procedure 3 that fully controls the variation of the different quantities leading to the approximated equilibrium state when the condition (78) is satisfied. Note that as given above, that procedure depends on the stiffened gas EOS, but it can easily extendable to other EOS as far as we may express the energy as a direct function of the pressure and density.

4. Procedure 4: in that last procedure, we want to define an iterative process that does not depend on (or that is not restricted to) a given EOS so explicitly as it is done in the procedure above. The starting point lies in a new formulation of the initial equations in terms of the physical variables.

We recall that we initially aim to solve approximately system (25). That system may be rewritten in terms of the physical variables $P_{m}, P_{I}, \alpha_{m}, \rho_{m}, c_{m, I}^{2}$, for $m=g, l$, where

$$
c_{m, I}^{2} \stackrel{\text { def }}{=} \frac{\frac{P_{\boldsymbol{I}}}{\rho_{m}^{2}}-\left.\frac{\partial e_{m}}{\partial \rho_{m}}\right|_{P_{m}}}{\left.\frac{\partial e_{m}}{\partial P_{m}}\right|_{\rho_{m}}}
$$

stands for the speed of sound at the interface of the fluid $m$. Assuming that the EOS writes as

$$
e_{m}=e_{m}\left(\rho_{m}, P_{m}\right)
$$

we have

$$
\frac{\partial e_{m}}{\partial t}=\left.\frac{\partial e_{m}}{\partial P_{m}}\right|_{\rho_{m}} \frac{\partial P_{m}}{\partial t}+\left.\frac{\partial e_{m}}{\partial \rho_{m}}\right|_{P_{m}} \frac{\partial \rho_{m}}{\partial t} .
$$

Using $E_{m}=e_{m}+\frac{1}{2} u_{m}^{2}$, and the fact that $u_{m}$ and $\left(\alpha_{m} \rho_{m}\right)$ remain constant during the process, we end with the following system of time partial equations, equivalent to system (25)

$$
\left\{\begin{array}{l}
\frac{\partial P_{g}}{\partial t}=-\frac{\rho_{g} c_{g, I}^{2}}{\alpha_{g}} \frac{\partial \alpha_{g}}{\partial t}, \\
\frac{\partial P_{l}}{\partial t}=\frac{\rho_{l} c_{l, I}^{2}}{\alpha_{l}} \frac{\partial \alpha_{l}}{\partial t} .
\end{array}\right.
$$

Integrating those equations on the time interval $\left[T_{0}, T^{*}\right]$, we get

$$
\left\{\begin{aligned}
\int_{T_{0}}^{T^{*}} \frac{\partial P_{g}}{\partial t} d t=P_{g}^{*}-P_{g}^{(0)} & =-\int_{T_{0}}^{T^{*}} \frac{\rho_{g} c_{g, I}^{2}}{\alpha_{g}} \frac{\partial \alpha_{g}}{\partial t} d t \\
& =-\int_{\alpha_{g}^{(0)}}^{\alpha_{g}^{*}} \frac{\rho_{g} c_{g, I}^{2}}{\alpha_{g}} d \alpha_{g} \\
\int_{T_{0}}^{T^{*}} \frac{\partial P_{l}}{\partial t} d t=P_{l}^{*}-P_{l}^{(0)} & =\int_{T_{0}}^{T^{*}} \frac{\rho_{l} c_{l, I}^{2}}{\alpha_{l}} \frac{\partial \alpha_{g}}{\partial t} d t \\
& =\int_{\alpha_{g}^{(0)}}^{\alpha_{g}^{*}} \frac{\rho_{l} c_{l, I}^{2}}{\alpha_{l}} d \alpha_{g},
\end{aligned}\right.
$$

i.e.

$$
\left\{\begin{array}{l}
P_{g}^{*}-P_{g}^{(0)}=-\int_{\alpha_{g}^{(0)}}^{\alpha_{g}^{*}} \frac{\rho_{g} c_{g, I}^{2}}{\alpha_{g}} d \alpha_{g} \\
P_{l}^{*}-P_{l}^{(0)}=\int_{\alpha_{g}^{(0)}}^{\alpha_{g}^{*}} \frac{\rho_{l} c_{l, I}^{2}}{\alpha_{l}} d \alpha_{g} .
\end{array}\right.
$$

It now remains to approximate the integrals of the right hand sides. Many approximations are available. We choose the following (simplest) one $(m=g, l)$

$$
\int_{\alpha_{g}^{(0)}}^{\alpha_{g}^{*}} \frac{\rho_{m} c_{m, I}^{2}}{\alpha_{m}} d \alpha_{g} \simeq\left[\left(\frac{\rho_{m} c_{m, I}^{2}}{\alpha_{m}}\right)^{(0)}\right] \Delta \alpha_{g}^{*}
$$


That is not the most accurate approximation, but its simple expression allows an easier description of the iterative algorithm. Let us replace now the indexes $(0)$ and $*$ by $(k)$ and $(k+1)$ respectively. We then get

$$
\left\{\begin{array}{l}
P_{g}^{(k+1)}-P_{g}^{(k)}=-\left(\frac{\rho_{g} c_{g, I}^{2}}{\alpha_{g}}\right)^{(k)} \Delta \alpha_{g}^{(k+1)} \\
P_{l}^{(k+1)}-P_{l}^{(k)}=\left(\frac{\rho_{l} c_{l, I}^{2}}{\alpha_{l}}\right)^{(k)} \Delta \alpha_{g}^{(k+1)}
\end{array}\right.
$$

where $\Delta \alpha_{g}^{(k+1)}$ is defined by

$$
\Delta \alpha_{g}^{(k+1)}=\varepsilon^{(k+1)} \frac{\Delta P^{(k)}}{C_{g}^{(k)}+C_{l}^{(k)}},
$$

with $\left.\left.\varepsilon^{(k+1)} \in\right] 0,1\right]$, and for $m=g, l$

$$
C_{m}^{(k)}=\left(\frac{\rho_{m} c_{m, I}^{2}}{\alpha_{m}}\right)^{(k)}
$$

This choice is motivated by the fact that if $(k+1)$ corresponds to the final step i.e.

$$
P_{g}^{(k+1)}=P_{l}^{(k+1)}=P_{I}^{(k+1)}=P^{*}
$$

then the definition of $\Delta \alpha_{g}^{(k+1)}$ is necessarily the definition given by in (100) with $\varepsilon^{(k+1)}=1$.

We may now describe the process as follows: given two positive arbitrarily small parameters $\varepsilon_{\alpha}$ and $\varepsilon_{P}$, and for each interior computational cell satisfying either

$$
\min \left(\alpha_{g}^{(k)}, \alpha_{l}^{(k)}\right)>\varepsilon_{\alpha}
$$

or

$$
\frac{\left|\Delta P^{(k)}\right|}{P_{I}^{(k)}}>\varepsilon_{P}
$$

we compute $\Delta \alpha_{g}^{(k+1)}$ by (100) where $\varepsilon^{(k+1)}$ is equal to $(1 / 2)^{n}$ where $n$ denotes here the number of times the initial value of $\Delta \alpha_{g}^{(k+1)}$ is divided by two if one of the following two conditions is not satisfied (condition on admissible value for $\alpha_{g}^{(k+1)}$ )

$$
\alpha_{g}^{(k+1)} \stackrel{\text { def }}{=} \alpha_{g}^{(k)}+\Delta \alpha_{g}^{(k+1)} \in[0,1],
$$

or (condition on monotonicity of the absolute pressure variation)

$$
\left|\Delta P^{(k+1)}\right| \leq\left|\Delta P^{(k)}\right|
$$

In fact, the definition of $\Delta \alpha_{g}^{(k+1)}$ given by (100) with $\varepsilon^{(k+1)}=1$ is valid if

$$
\frac{\left|\Delta P^{(k)}\right|}{C_{g}^{(k)}+C_{l}^{(k)}}<\min \left(\alpha_{g}^{(k)}, \alpha_{l}^{(k)}\right) .
$$

The iterative process stops if the relative pressure variation satifies

$$
\frac{\left|\Delta P^{(k)}\right|}{P_{I}^{(k)}} \leq \varepsilon_{P} .
$$

In such a case, as it has been done in the previous procedures described above, we update the conservative variables $\left(\alpha_{g}\right)$ and $\left(\alpha_{m} \rho_{m} E_{m}\right)$, for $m=g, l$

$$
\begin{cases}\left(\alpha_{g}\right) & =\alpha_{g}^{(k+1)} \\ \left(\alpha_{m} \rho_{m} E_{m}\right) & =\alpha_{m}^{(k+1)} \rho_{m}^{(k+1)}\left(e_{m}^{(k+1)}+\frac{1}{2}\left(u_{m}^{(0)}\right)^{2}\right),\end{cases}
$$


where, for $m=g, l$

$$
\left\{\begin{array}{l}
\rho_{m}^{(k+1)}=\left(\alpha_{m} \rho_{m}\right)^{(0)} / \alpha_{m}^{(k+1)} \\
e_{m}^{(k+1)}=e_{m}\left(P_{m}^{(k+1)}, \rho_{m}^{(k+1)}\right) .
\end{array}\right.
$$

We thus have defined a simple iterative process which can be used with more general EOS than the stiffened gas EOS. It is moreover almost a direct method due to the definition of the volume fraction variation.

\section{$3 \quad$ Numerical tests}

We want to illustrate the use of those procedures by performing the two following numerical test cases

1. the water faucet test case of Ransom [8],

2. the air/water shock tube test as given by in [11] (pp. 448-450).

\subsection{Water faucet test}

Let us consider a vertical tube of 12 meters length. It is initially filled with water and surrounded by water vapour with a pressure equal to $10^{5} \mathrm{~Pa}$. At the top of that tube, the liquid velocity is set to $10 \mathrm{~m} / \mathrm{s}$ with a volume fraction $\alpha_{l}$ equal to 0.8 . The bottom of the tube is open to the ambiant pressure $\left(10^{5} \mathrm{~Pa}\right.$. $)$. At $T=0$, the tube is filled with a uniform column of water at velocity $10 \mathrm{~m} / \mathrm{s}$, surrounded by stagnant water vapour with a volume fraction $\alpha_{g}$ equal to 0.2 . Under the effect of gravity, the liquid is accelerated and the void profile propagates along the tube.

We suppose that each fluid is governed by the stiffened gas equation of state, where

$$
\gamma_{g}=1.4, P_{g, \infty}=0 \mathrm{~Pa}, \rho_{g}=1.0 \mathrm{~kg} / \mathrm{m}^{3},
$$

for the gaz which is considered as a perfect gas here, and

$$
\gamma_{l}=4.0, P_{l, \infty}=6 \times 10^{5} \mathrm{~Pa}, \rho_{l}=10^{3} \mathrm{~kg} / \mathrm{m}^{3},
$$

for the liquid fluid.

The initial velocity of the gaz is zero. We therefore use the 7 equations model, where each phase has its own velocity, combined with the pressure relaxation correction step. We here model that problem where each fluid behaves as incompressible fluid, as they were compressible. We also add the effect of gravitation in the right hand sides of the 7 equations model.

Two series of tests are performed with respectively 104 and 520 discretization nodes, and using the different pressure relaxation procedures described in the previous sections

i Proc0 : direct procedure (new, cf. 2.2.4)

ii Proc1: procedure 1 (iterative process),

iii Proc2 : procedure 2 (iterative process),

iv Proc3 : procedure 3 (new, iterative process),

v Proc4 : procedure 4 (new, iterative process, approach given in terms of physical variables).

For each of those procedures, we compare the different approximated solutions at $t=0.4 \mathrm{~s}$.. For that problem, we are able to get an analytic solution [4] which writes as

$$
\left\{\begin{array}{llll}
\alpha_{g}(x) & =1-\alpha_{l} u_{l}(x) / \sqrt{2 g x+u_{l}(x)^{2}}, & & \text { if } x \leq u_{l}(x) t+g t^{2} / 2, \\
& =1-\alpha_{l}, & & \text { otherwise }
\end{array}\right.
$$

where $\alpha_{l}=0.8$ is the initial value (at $t=0$ ) of the volume fraction of the liquid phase and $g$ is the gravity constant $g=9.81$. 
For the different tests we have performed, the test parameters are set by

$$
\varepsilon_{0}=1 / 2, \varepsilon_{\alpha}=10^{-8}, \varepsilon_{P}=10^{-8} .
$$

In Figure 1 (resp. 2), we give the air volume fraction at $t=0,4$ s., for the 104 nodes test (resp. 520 nodes test). Because of the use of the HLL solver, the approximated solution is very diffusive, but with 520 nodes, the result is quite correct.

Table 1 gives the computational costs (on a HP-UX 9000/782 station) of the different tests. As it shows, the direct procedure, Proc. 0, is of course the more efficient, but Proc. 4 is almost direct and is the most competitive (and robust) of all existing iterative procedures used for those tests.

\begin{tabular}{||c||r|r|r|r|r||}
\hline \hline problem size & Proc. 0 & Proc. 1 & Proc. 2 & Proc. 3 & Proc. 4 \\
\hline 104 & $18.51 \mathrm{~s}$. & $46.63 \mathrm{~s}$. & $73.05 \mathrm{~s}$. & $77.24 \mathrm{~s}$. & $24.35 \mathrm{~s}$. \\
\hline \hline 520 & $442.47 \mathrm{~s}$. & $1165.58 \mathrm{~s}$. & $1525.13 \mathrm{~s}$. & $1960.59 \mathrm{~s}$. & $616.92 \mathrm{~s}$. \\
\hline \hline
\end{tabular}

Table 1: CPU costs for the water faucet problem.

\subsection{Air/water shock tube problem}

Let us consider the shock tube with 1 meter length, filled on the left by liquid water at hight pressure and on the right by air. That problem also possesses an analytical solution. Here again, both fluid are considered compressible and governed by the stiffened gas EOS. The initial data are set as

$$
\left\{\begin{array}{lll}
\rho_{l}=10^{3} \mathrm{~kg} / \mathrm{m}^{3}, & P_{l}=10^{9} \mathrm{~Pa}, & u_{l}=0 \mathrm{~m} / \mathrm{s} \\
\gamma_{l}=4.4, & P_{l, \infty}=610^{8} \mathrm{~Pa}, & \alpha_{l}=1-\varepsilon
\end{array}\right.
$$

if $x<0.7$, and

$$
\left\{\begin{array}{lll}
\rho_{g}=50 \mathrm{~kg} / \mathrm{m}^{3}, & P_{g}=10^{5} \mathrm{~Pa}, & u_{g}=0 \mathrm{~m} / \mathrm{s}, \\
\gamma_{g}=1.4, & P_{g, \infty}=0 \mathrm{~Pa}, & \alpha_{g}=1-\varepsilon,
\end{array}\right.
$$

if $x \geq 0.7$, where $\varepsilon=10^{-8}$. For that test problem, we use the 7 equations model with both velocity and pressure relaxation processes.

Here again, two series of tests have been performed with 101 and 1001 discretization nodes and we compare the results obtained by using the different pressure relaxation procedures as in the water faucet test problem. The test parameters used for those numerical tests are set as:

$$
\varepsilon_{0}=1 / 2, \varepsilon_{\alpha}=10^{-8}, \varepsilon_{P}=1.510^{1} .
$$

The approximated solutions at time $t=229.010^{-6} \mathrm{~s}$. of the 101 nodes (resp. 1001 nodes) test are about the same (cf. figures 3-8 (101 nodes) and figures 9-14 (1001 nodes)), except for the one obtained with Proc. 4: as a matter of fact, that solution is more accurate as it is shown for the mixture density $\bar{\rho}$ in Fig. 4 , as for the equilibrium velocity in Fig. 5) and at last the interfacial pressure in Fig. 7).

Denoting by $P_{e x}$ the analytical pressure and defining the relative pressure error $\epsilon_{P}$ by

$$
\epsilon_{P} \stackrel{\text { def }}{=} \frac{P_{e x}-P_{I}}{P_{e x}}
$$

we indeed see that Proc. 4 gives a better resolution as it is shown in table 2 (101 nodes) and table 3 (1001 nodes).

\begin{tabular}{||c||r|r|r|r|r||}
\hline \hline$\varepsilon_{P}$ & Proc. 0 & Proc. 1 & Proc. 2 & Proc. 3 & Proc. 4 \\
\hline min & $1.38 \mathrm{e}-6$ & $1.65 \mathrm{e}-6$ & $1.37 \mathrm{e}-6$ & $1.36 \mathrm{e}-6$ & $5.13 \mathrm{e}-8$ \\
\hline \hline $\max$ & 104.62 & 111.07 & 104.60 & 104.43 & 55.37 \\
\hline \hline
\end{tabular}

Table 2: Relative pressure error - Air/water shock tube problem (101 nodes). 


\begin{tabular}{||c||r|r|r|r|r||}
\hline \hline$\varepsilon_{P}$ & Proc. 0 & Proc. 1 & Proc. 2 & Proc. 3 & Proc. 4 \\
\hline $\min$ & 0.0 & 0.0 & 0.0 & 0.0 & 0.0 \\
\hline \hline $\max$ & 110.52 & 118.88 & 109.77 & 109.72 & 87.54 \\
\hline \hline
\end{tabular}

Table 3: Relative pressure error - Air/water shock tube problem (1001 nodes).

In Table 4, the CPU costs for those tests are given.

\begin{tabular}{||c||r|r|r|r|r||}
\hline \hline Problem size & Proc. 0 & Proc. 1 & Proc. 2 & Proc. 3 & Proc. 5 \\
\hline 101 & $1.67 \mathrm{~s}$. & $2.86 \mathrm{~s}$. & $3.29 \mathrm{~s}$. & $5.41 \mathrm{~s}$. & $2.00 \mathrm{~s}$. \\
\hline \hline 1001 & $133.42 \mathrm{~s}$. & $202.85 \mathrm{~s}$. & $224.48 \mathrm{~s}$. & $325.37 \mathrm{~s}$. & $158.08 \mathrm{~s}$. \\
\hline \hline
\end{tabular}

Table 4: CPU costs for the air/water shock tube problem.

\section{Conclusion}

Several pressure relaxation procedures are developed and compared in terms of accuracy and computational efficiency in the context of a compressible hyperbolic two-phase flow model.

Among those procedures, the one defined from the formulation given in terms of physical variables appears the most attractive, robust and low CPU consuming. It is particularly adapted to strong shocks problems, i.e. in situations where the pressure variation is high. In addition, that procedure gives a better accuracy than the others as it has been enhanced in the air/water shock tube problem.

Combined with a less diffusive Riemann solver than HLL, that improvement will be a good starting point for the good solution of multifluid flow problems and multiphase problems with interfaces. 


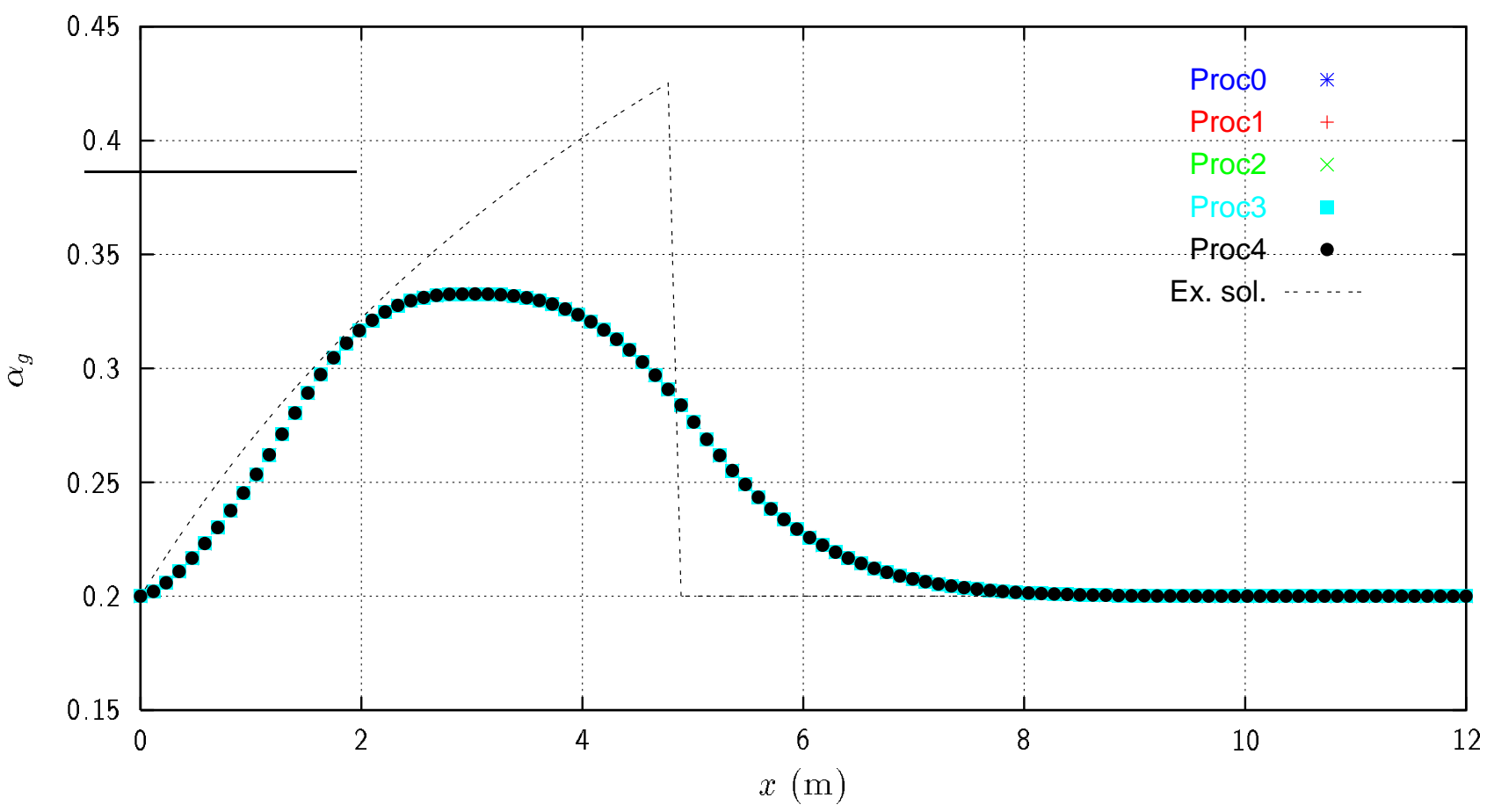

Figure 1: Void fractions at $t=0.4 \mathrm{~s}$. - Water faucet (104 nodes).

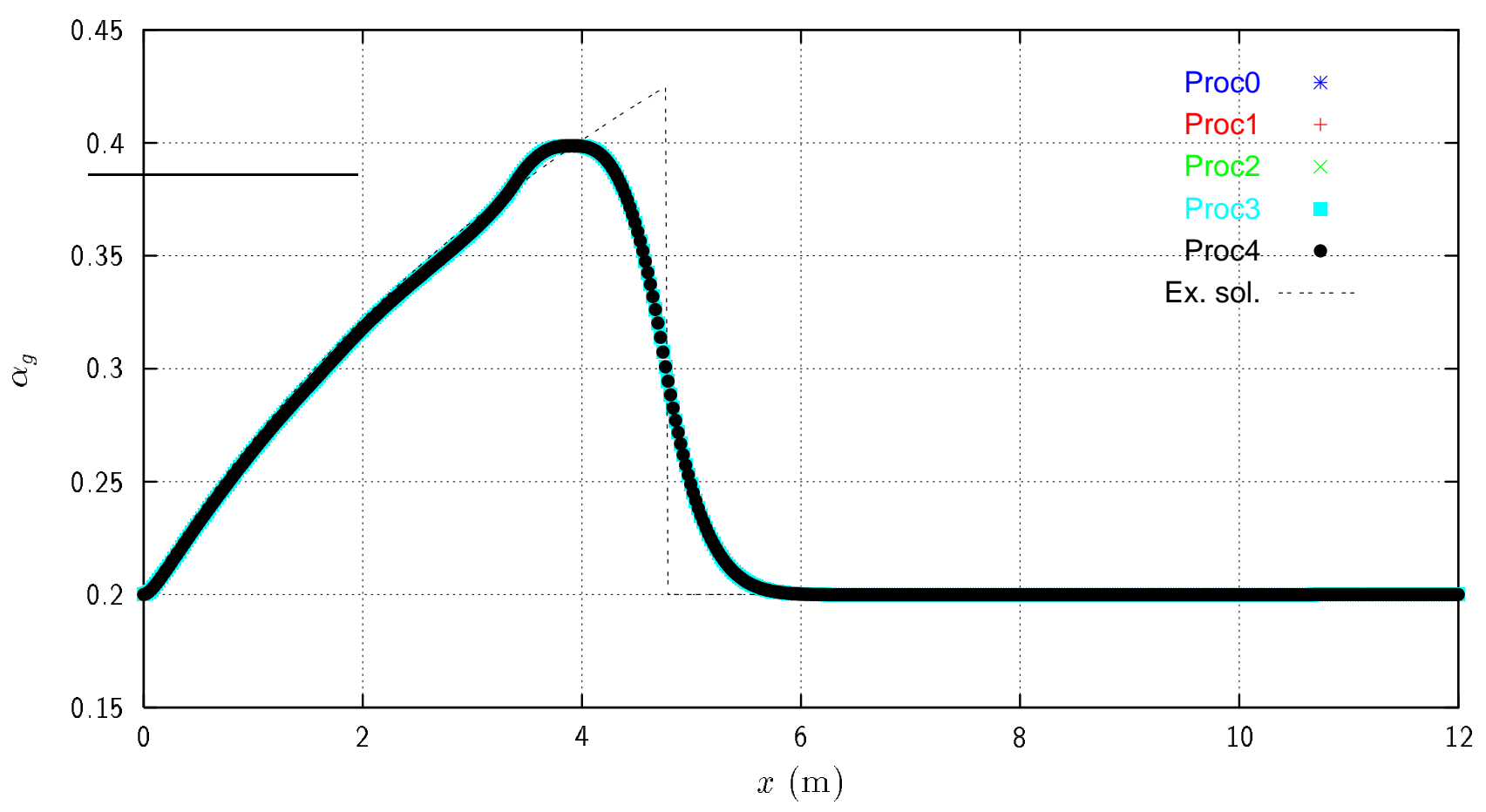

Figure 2: Void fractions at $t=0.4$ s.- Water faucet (520 nodes). 


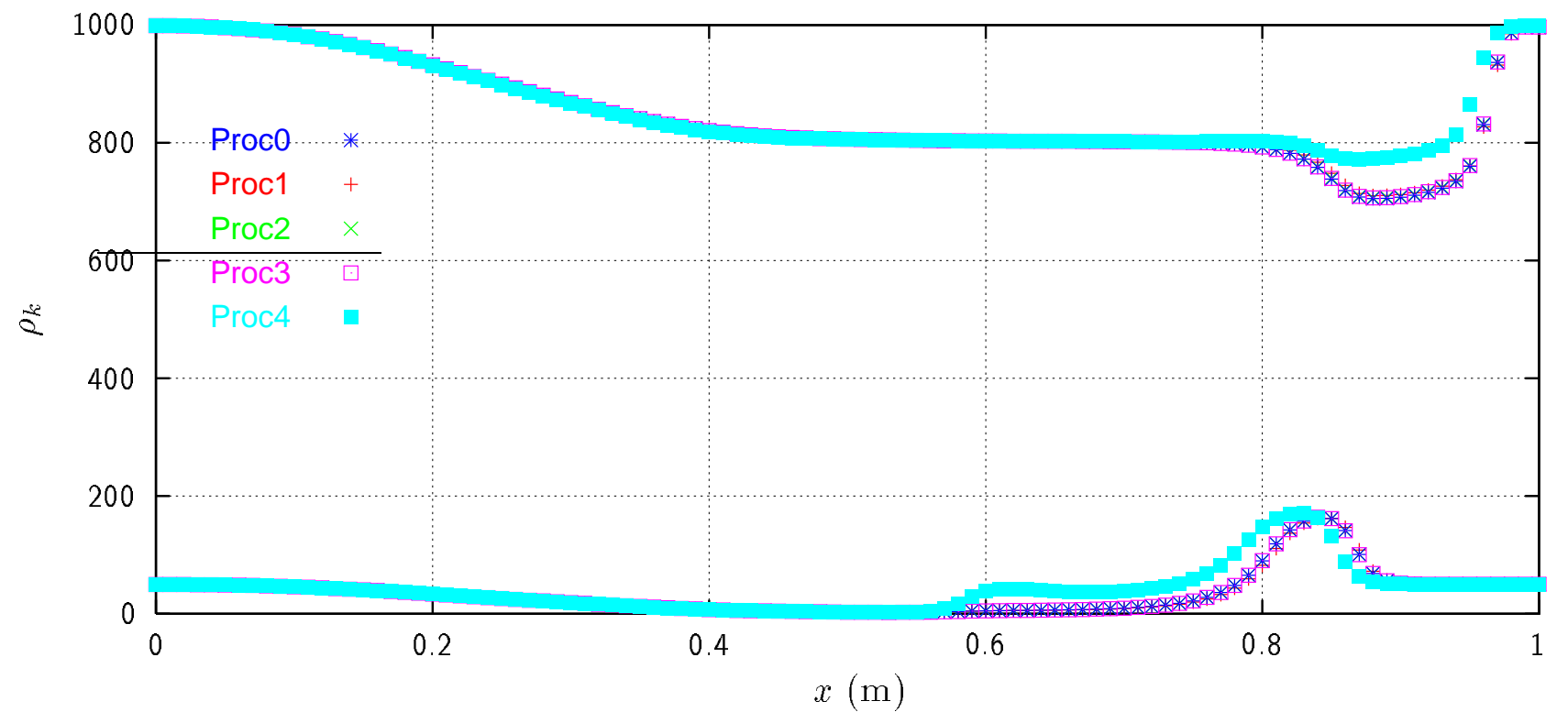

Figure 3: Phase densities at $t=229 \cdot 10^{-6}$ s. - Air/water shock tube (101 nodes).

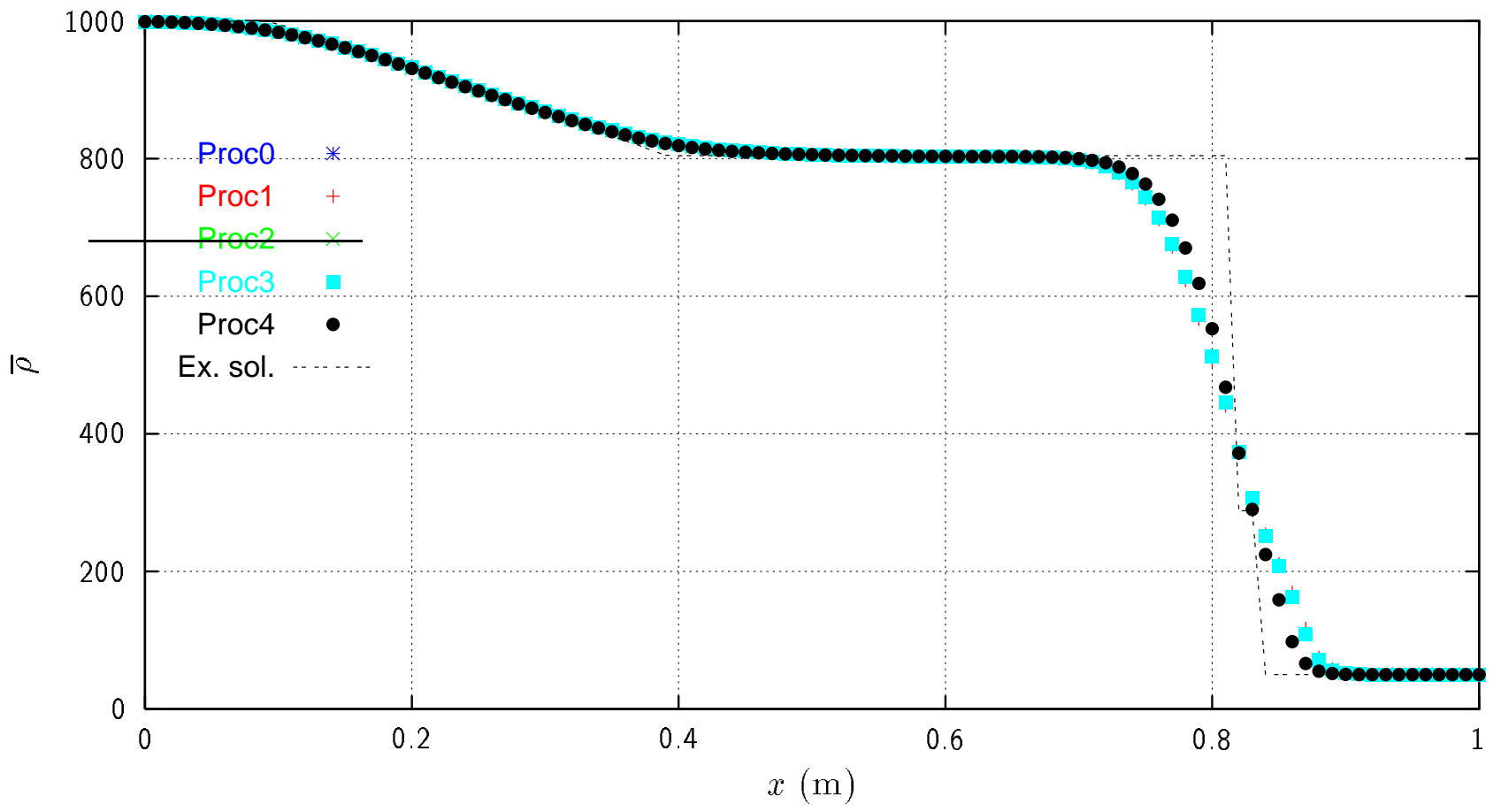

Figure 4: Mixture densities at $t=229 \cdot 10^{-6}$ s. - Air/water shock tube (101 nodes). 


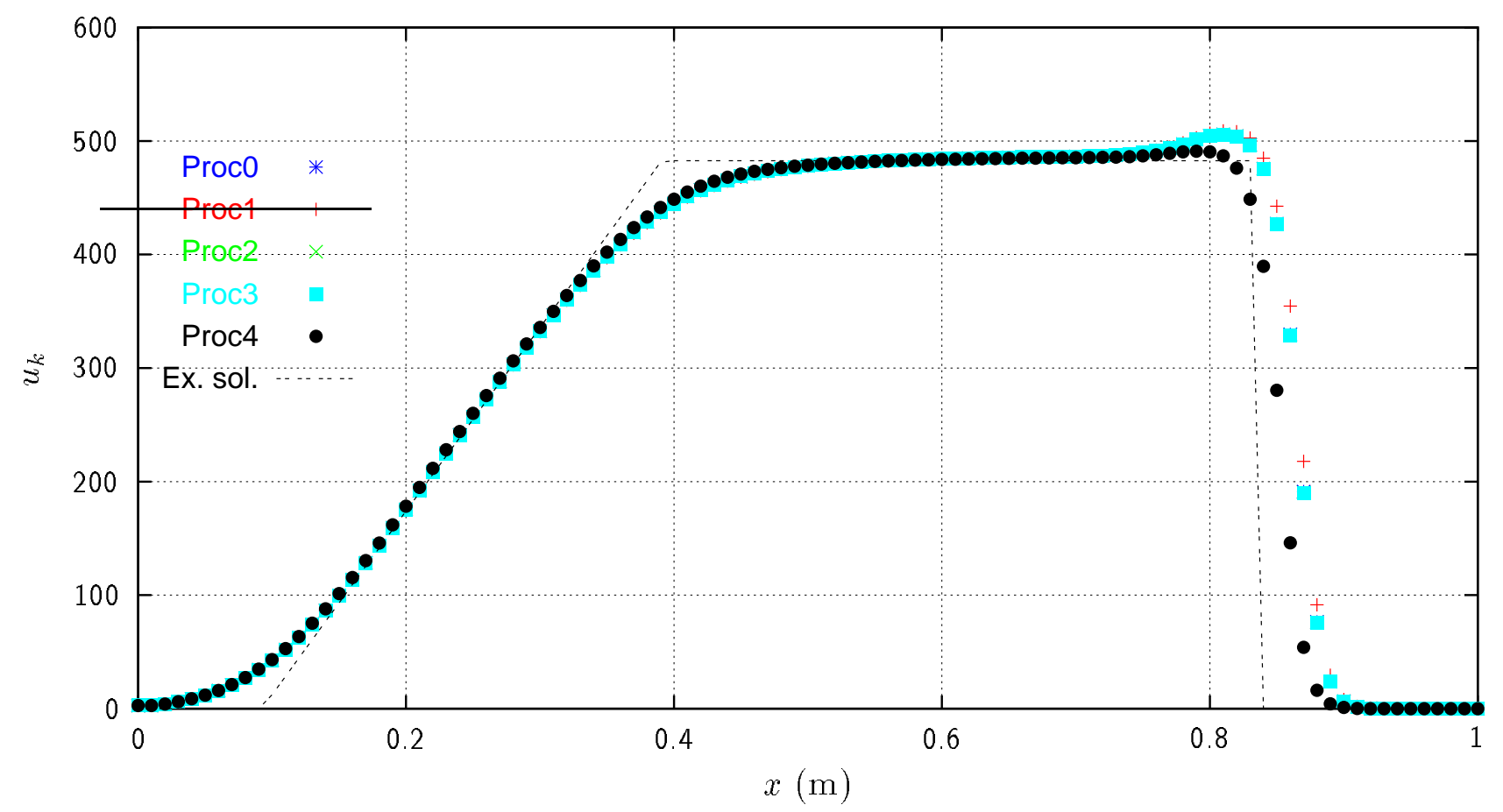

Figure 5: Equilibrium velocities at $t=224 \cdot 10^{-6} \mathrm{~s}$. - Air/water shock tube (101 nodes).

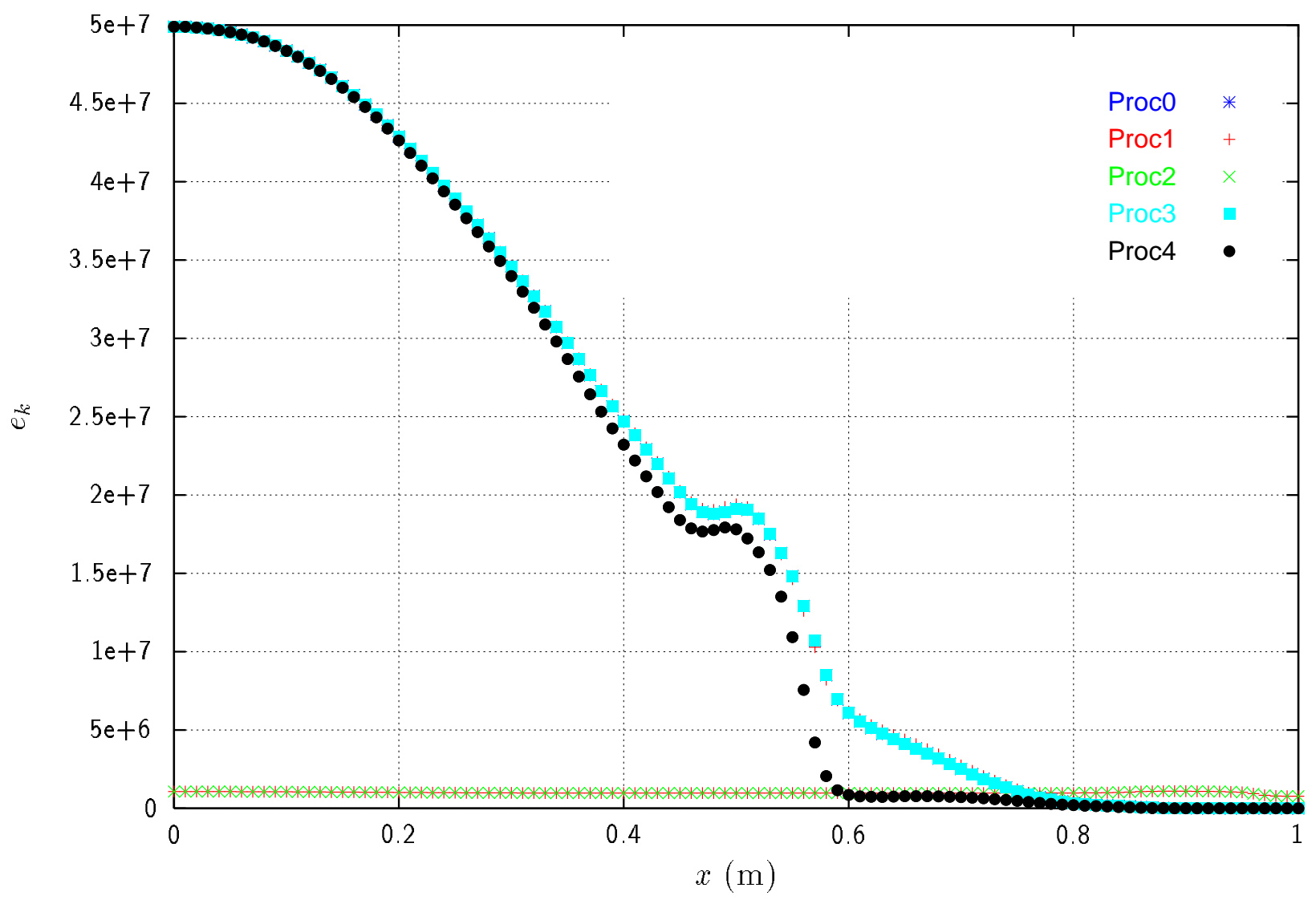

Figure 6: Phase internal energies at $t=224 \cdot 10^{-6} \mathrm{~s}$. - Air/water shock tube (101 nodes). 


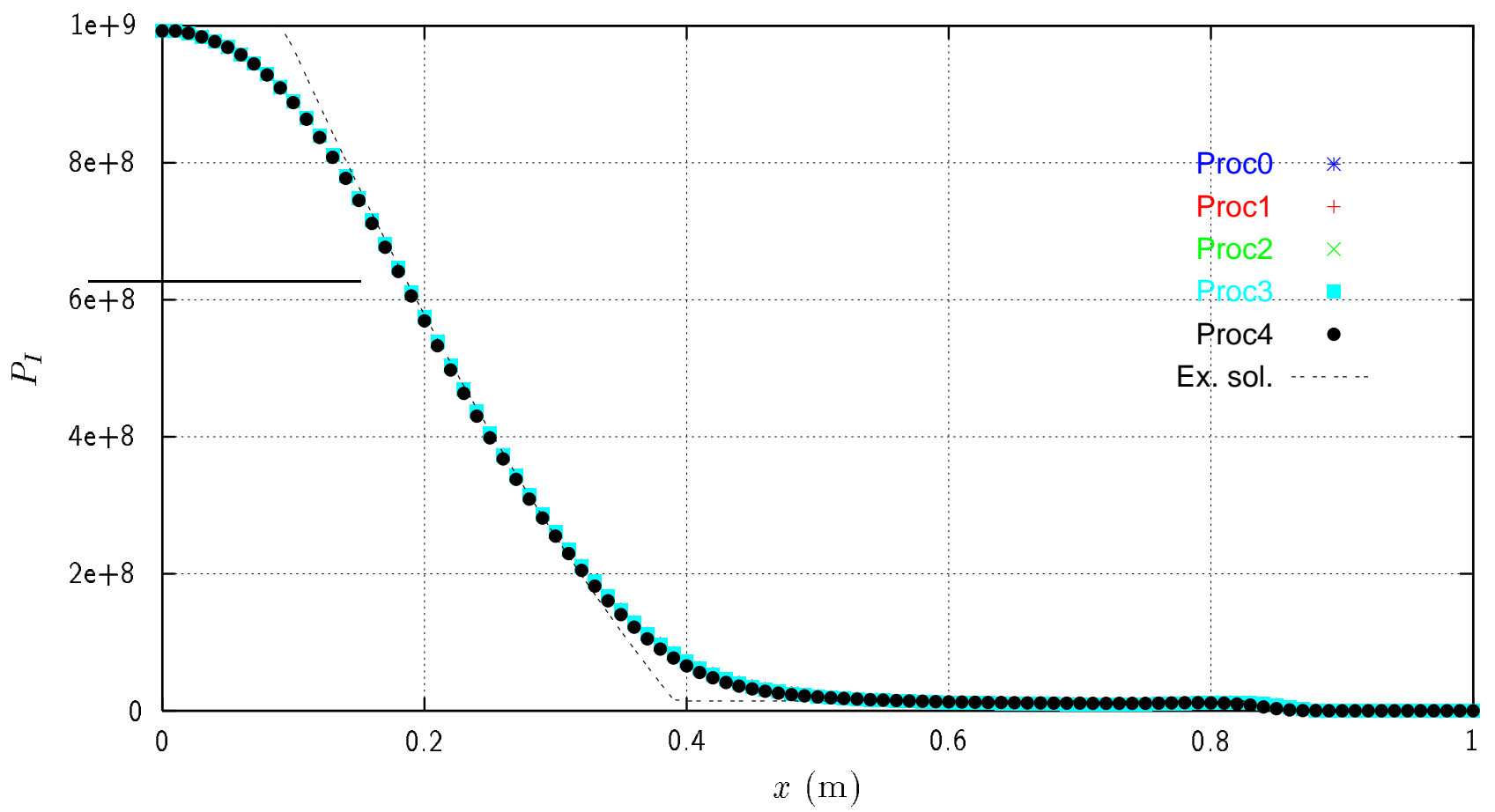

Figure 7: Interfacial pressures at $t=224 \cdot 10^{-6} \mathrm{~s}$.- Air/water shock tube (101 nodes).

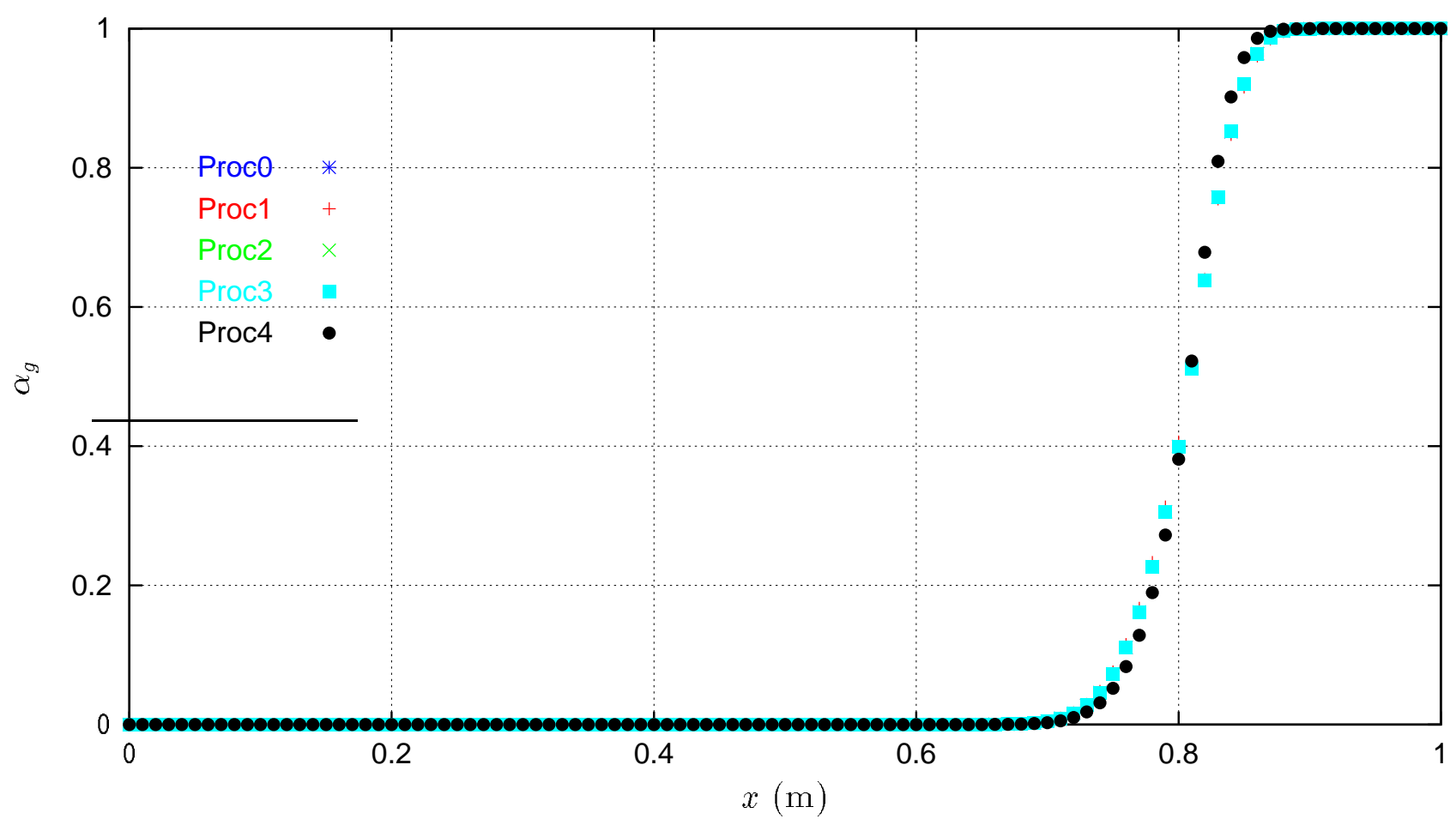

Figure 8: Void fractions at $t=224 \cdot 10^{-6} \mathrm{~s}$. - Air/water shock tube (101 nodes). 


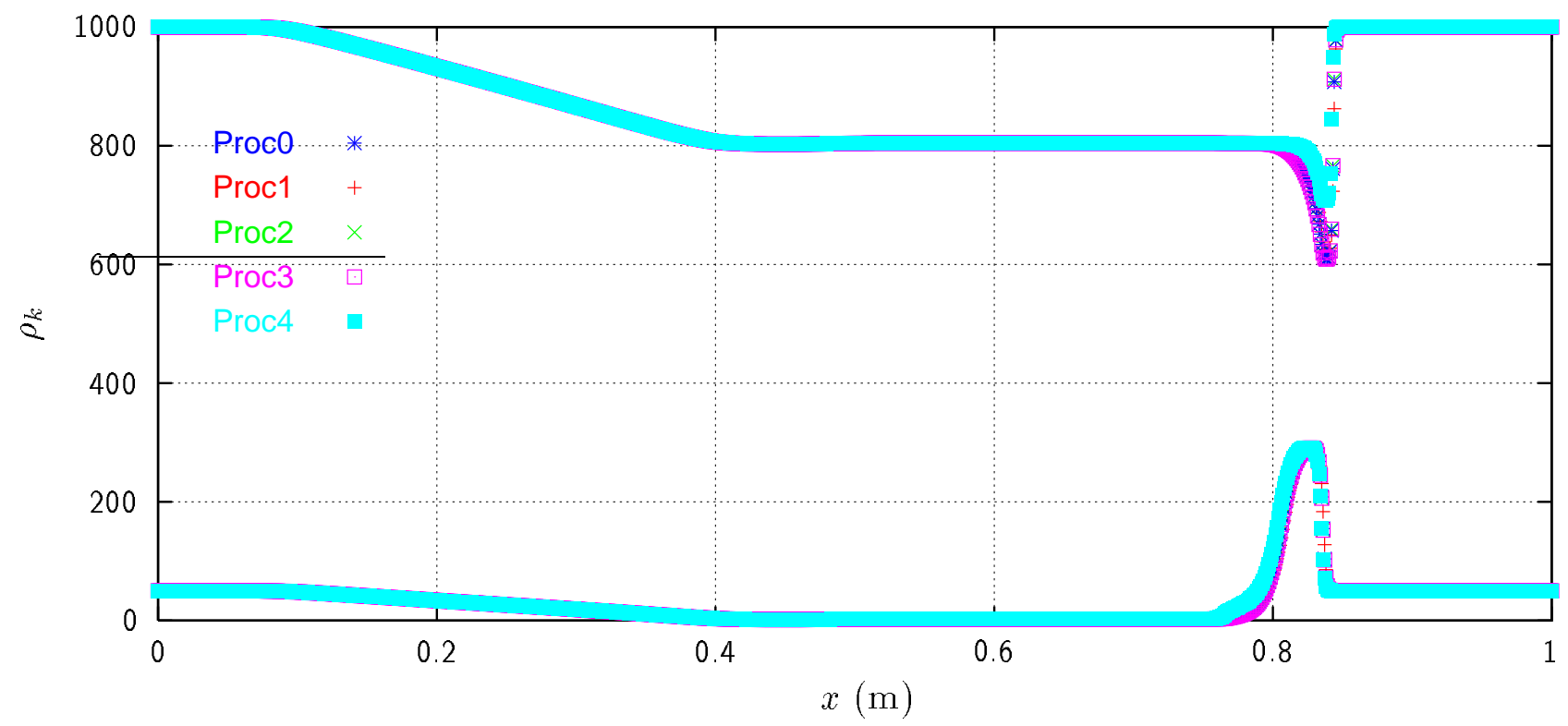

Figure 9: Phase densities at $t=229 \cdot 10^{-6} \mathrm{~s}$. - Air/water shock tube (1001 nodes).

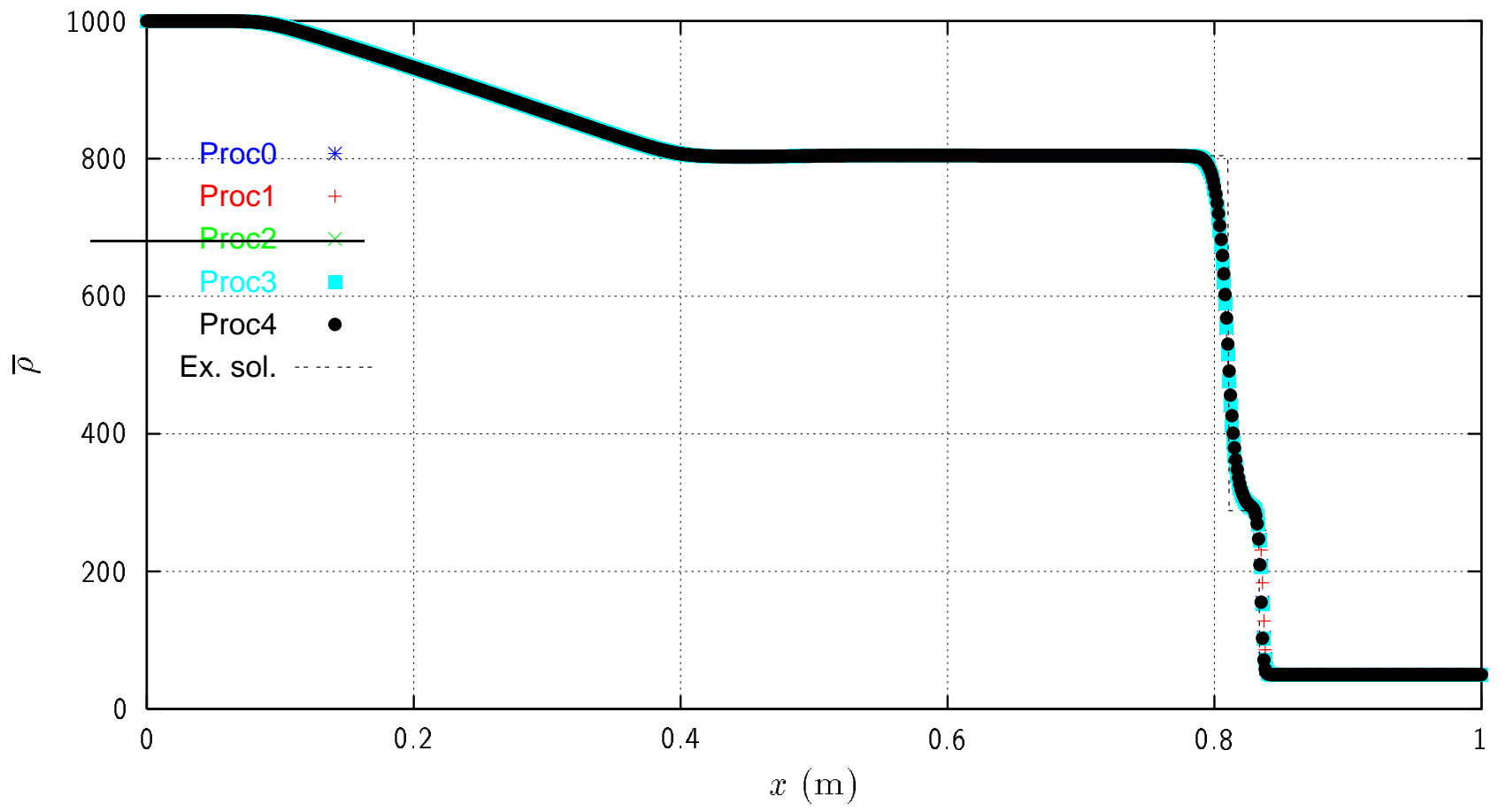

Figure 10: Mixture densities at $t=229 \cdot 10^{-6}$ s. - Air/water shock tube (1001 nodes). 


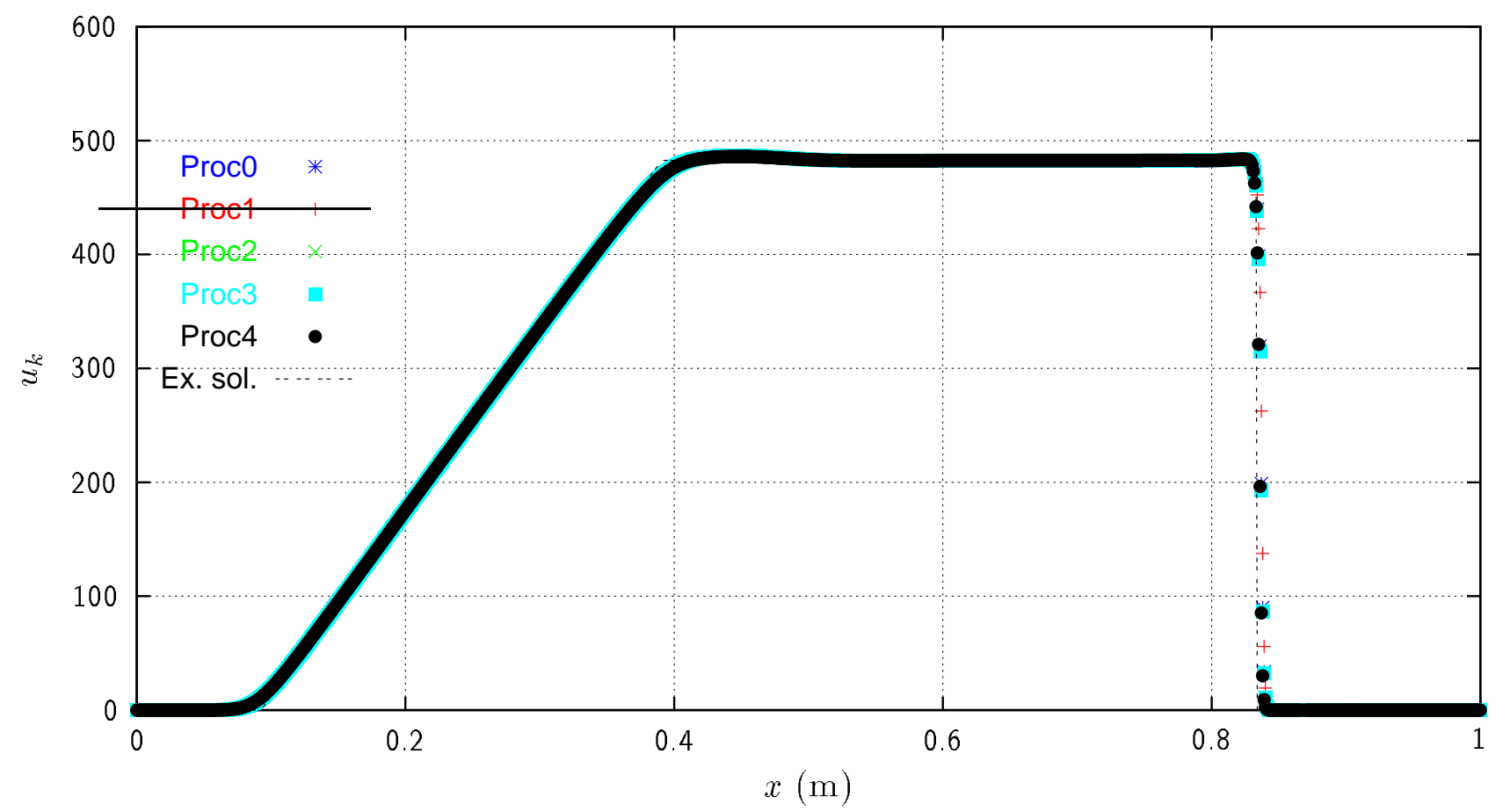

Figure 11: Equilibrium velocities at $t=224 \cdot 10^{-6} \mathrm{~s}$. - Air/water shock tube (1001 nodes).

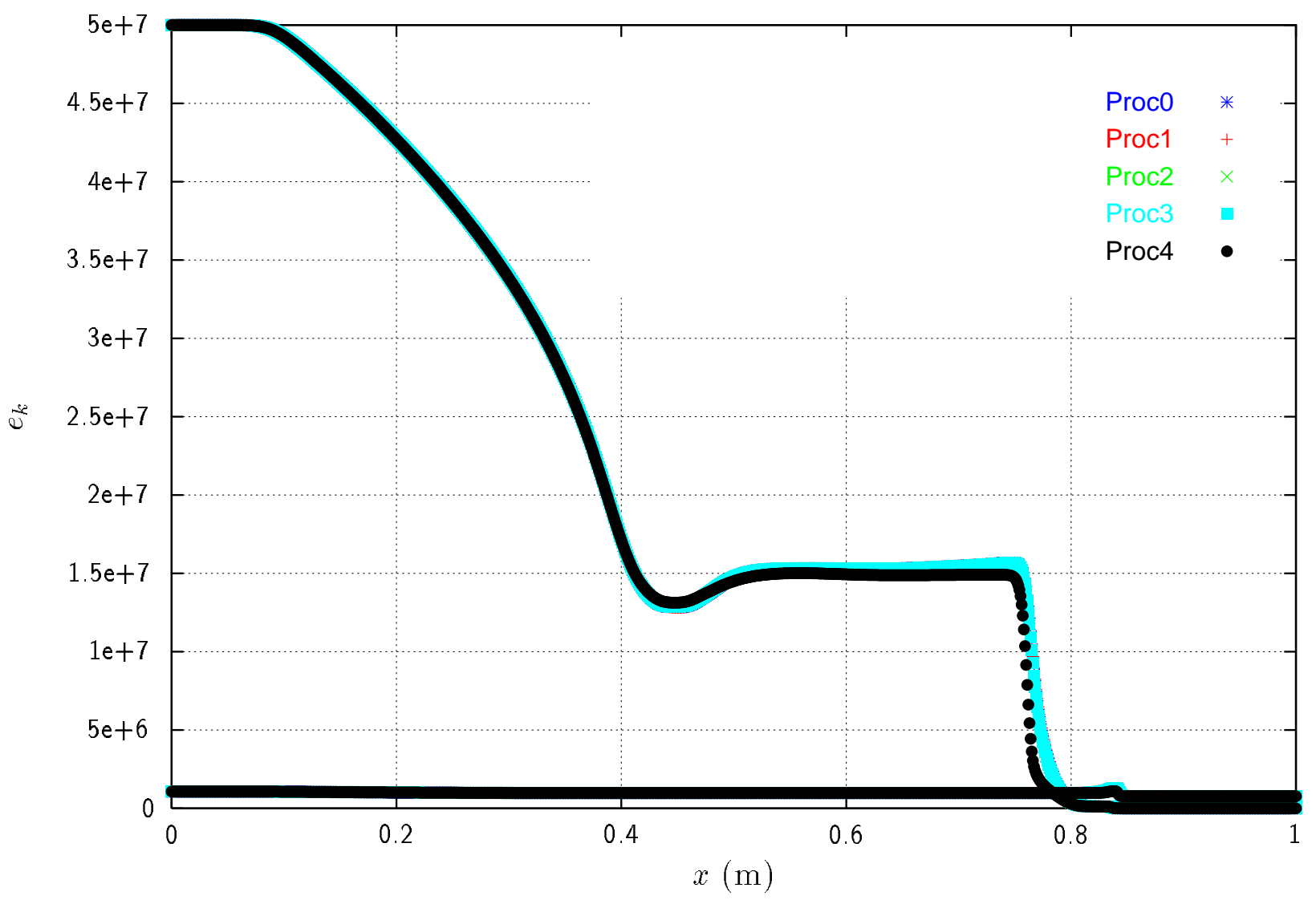

Figure 12: Phase internal energies at $t=224 \cdot 10^{-6} \mathrm{~s}$. - Air/water shock tube (1001 nodes). 


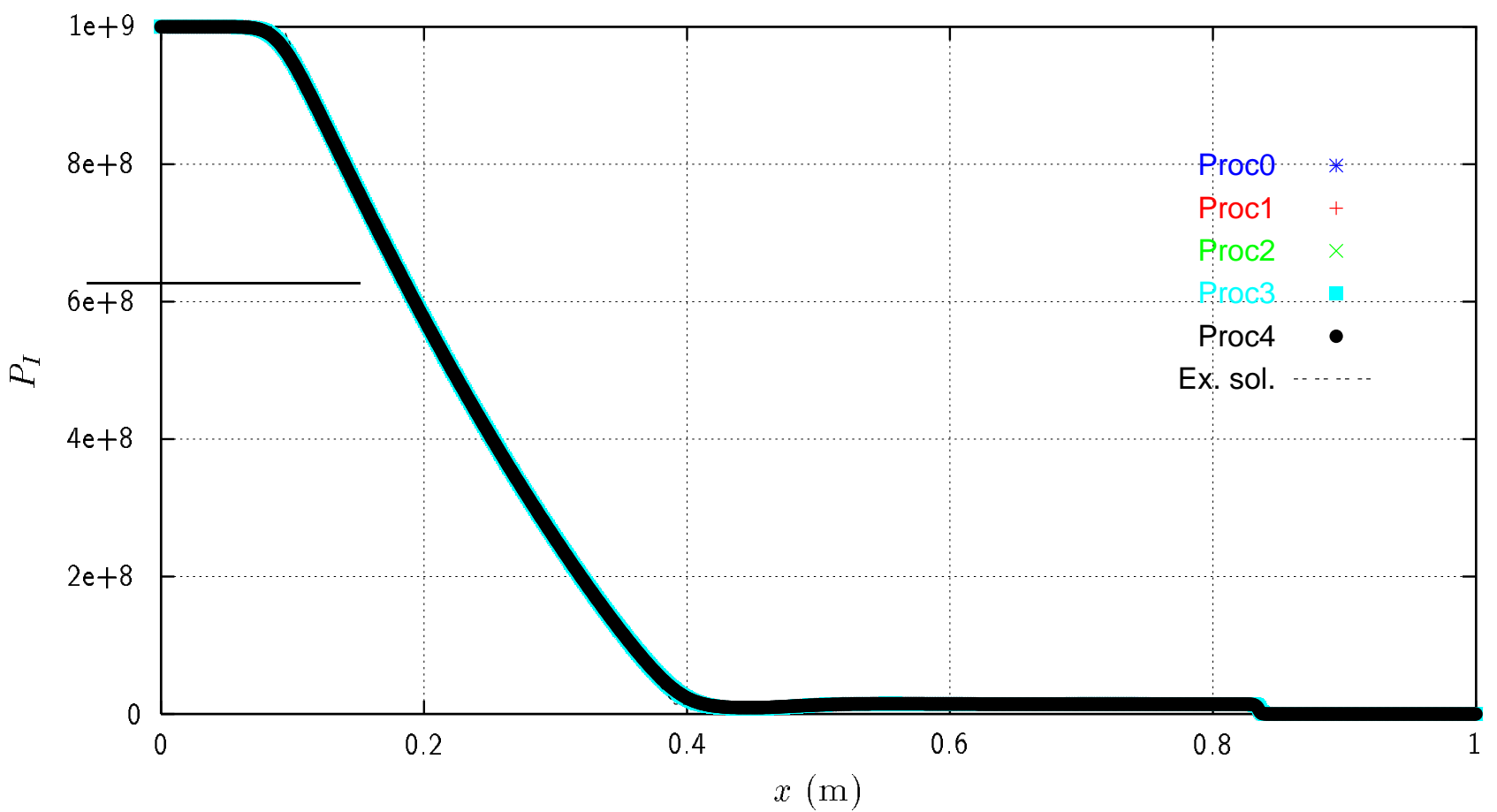

Figure 13: Interfacial pressures at $t=224 \cdot 10^{-6}$ s.- Air/water shock tube (1001 nodes).

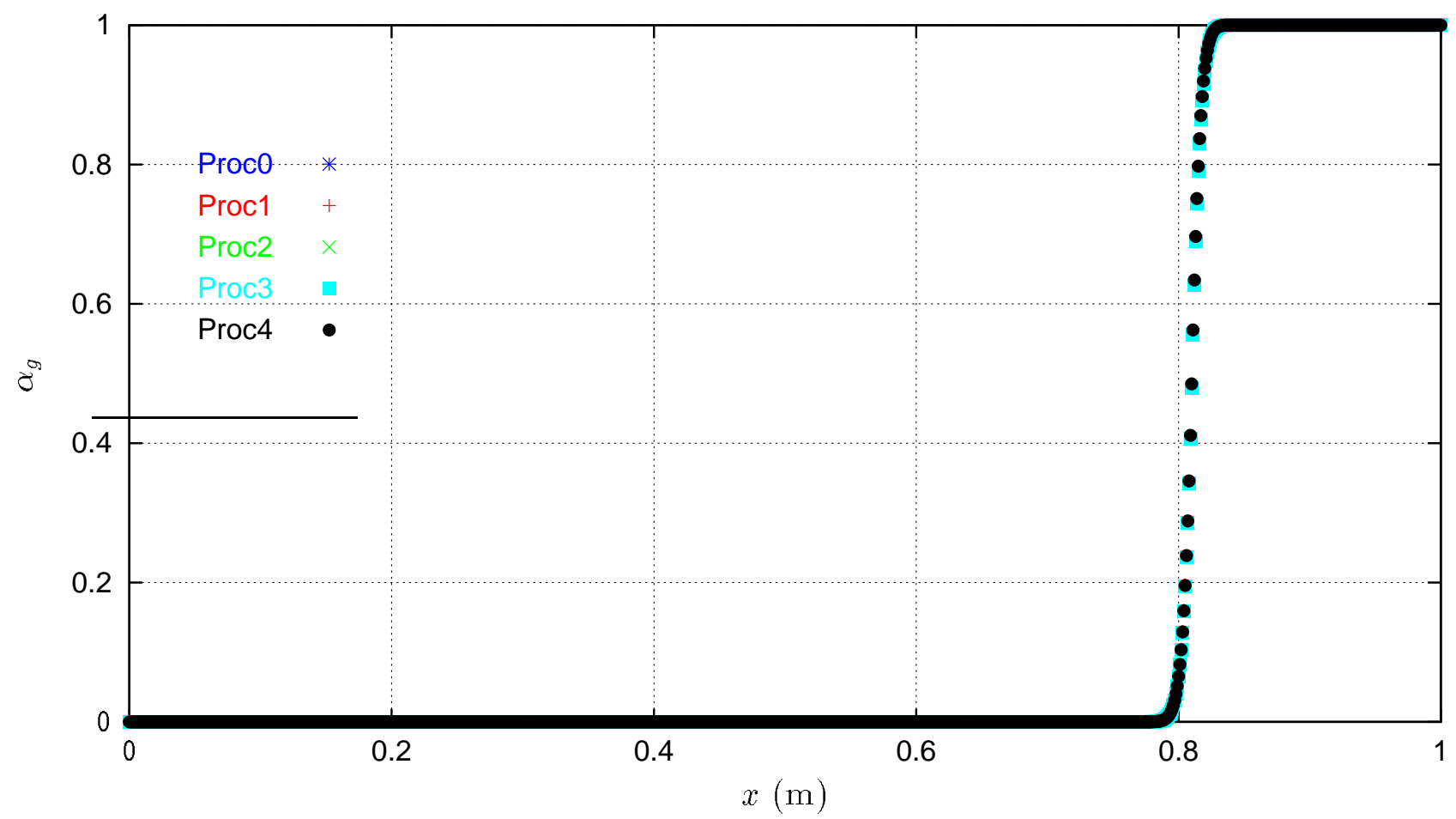

Figure 14: Void fractions at $t=224 \cdot 10^{-6} \mathrm{~s}$. - Air/water shock tube (1001 nodes). 


\section{References}

[1] M.R. Baer and J.W. Nunziato. A two-phase mixture theory for the deflagration-to-detonation transition (DDT) in reactive granular materials. Int. J. Multiphase Flows, 12(6):861-889, 1986.

[2] J.B. Bdzil, R. Menikoff, S.F. Son, A.K. Kapila, and D.S. Stewart. Two-phase modeling of DDT in granular materials: A critical examination of modeling issues. Physics of Fluids, 11(2):378-402, 1999.

[3] P.B. Butler, M.F. Lambeck, and H. Krier. Modeling of shock development and transition to detonation initiated by burning in porous propellant beds. Combust. Flame, 46:75-93, 1982.

[4] F. Coquel, K. El Amine, E. Godlewsky, B. Perthame, and P. Rascle. A numerical method using upwind schemes for the resolution of two-phase flows. Journal of Computational Physics, 136:272-288, 1997.

[5] S. K. Godunov, A. Zabrodine, M. Ivanov, A. Kraiko, and G. Prokopov. . In Résolution numérique des problèmes multidimensionnels de la dynamique des gaz. Éditions Mir, Moscow, 1979.

[6] A.K. Kapila, S.F. Son, J.B. Bdzil, R. Menikoff, and D.S. Stewart. Two-phase modeling of DDT : structure of the velocity relaxation zone. Physics of Fluids, 9(12):3885-3897, 1997.

[7] R. Menikoff and B.J. Plohr. The Riemann problem for fluid flow of real materials. Review of Modern Physics, 61(1):75-130, 1989.

[8] V. H. Ransom. Numerical benchmark tests. In Multiphase science and technology, volume 3. G. Hewitt, J. M. Delhaye, and N. Zuber, Hemisphere, Washington D.C., 1987.

[9] R. Saurel. Interfaces, detonation waves, cavitation and the multiphase Godunov method. In Kluver Academic Publishers, editor, International Conference to honour of Professor S.K. Godunov, the year of his 70th birthday, Oxford, October 1999 2000. Invited lecture in press in those proceedings.

[10] Richard Saurel. A multiphase model for compressible flows with interfaces, shoks, detonation waves and cavitations. Journal of Fluid Mechanics, 2000. accepted.

[11] Richard Saurel and Rémi Abgrall. A multiphase Godunov method for compressible multifluid and multiphase flows. Journal of Computational Physics, 150:425-467, 1999.

[12] G. Strang. On the construction and comparison of difference schemes. SIAM J. on Numer. Anal., 5(3):506$517,1968$. 


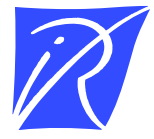

Unité de recherche INRIA Rocquencourt Domaine de Voluceau - Rocquencourt - BP 105 - 78153 Le Chesnay Cedex (France)

Unité de recherche INRIA Lorraine : LORIA, Technopôle de Nancy-Brabois - Campus scientifique 615, rue du Jardin Botanique - BP 101 - 54602 Villers-lès-Nancy Cedex (France)

Unité de recherche INRIA Rennes : IRISA, Campus universitaire de Beaulieu - 35042 Rennes Cedex (France)

Unité de recherche INRIA Rhône-Alpes : 655, avenue de l'Europe - 38330 Montbonnot-St-Martin (France)

Unité de recherche INRIA Sophia Antipolis : 2004, route des Lucioles - BP 93 - 06902 Sophia Antipolis Cedex (France)

INRIA - Domaine de Voluceau - Rocquencourt, BP 105 - 78153 Le Chesnay Cedex (France)

http://www.inria.fr

ISSN 0249-6399 